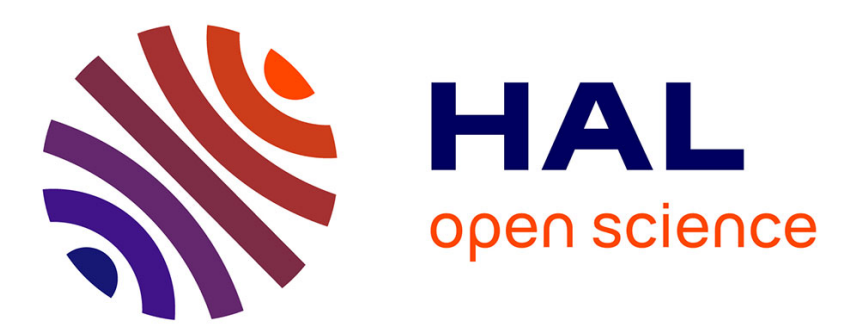

\title{
A semi-analytical approach to the study of an elastic cylinder confined in a cylindrical fluid domain subjected to small-amplitude transient motions
}

Cédric Leblond, Jean-François Sigrist, Bruno Auvity, Hassan Peerhossaini

\section{- To cite this version:}

Cédric Leblond, Jean-François Sigrist, Bruno Auvity, Hassan Peerhossaini. A semi-analytical approach to the study of an elastic cylinder confined in a cylindrical fluid domain subjected to small-amplitude transient motions. Journal of Fluids and Structures, 2008, 25, pp.134-154. 10.1016/j.jfluidstructs.2008.04.004 . hal-00403155

\section{HAL Id: hal-00403155 \\ https://hal.science/hal-00403155}

Submitted on 17 Apr 2018

HAL is a multi-disciplinary open access archive for the deposit and dissemination of scientific research documents, whether they are published or not. The documents may come from teaching and research institutions in France or abroad, or from public or private research centers.
L'archive ouverte pluridisciplinaire HAL, est destinée au dépôt et à la diffusion de documents scientifiques de niveau recherche, publiés ou non, émanant des établissements d'enseignement et de recherche français ou étrangers, des laboratoires publics ou privés. 


\title{
A semi-analytical approach to the study of an elastic circular cylinder confined in a cylindrical fluid domain subjected to small-amplitude transient motions
}

\author{
C. Leblond ${ }^{\mathrm{a}, 1}$, J.F. Sigrist ${ }^{\mathrm{a}, *}$, B. Auvity ${ }^{\mathrm{b}}$, H. Peerhossaini ${ }^{\mathrm{b}}$ \\ ${ }^{a}$ Service Technique et Scientifique, DCNS Propulsion, 44620 La Montagne, France \\ ${ }^{\mathrm{b}}$ Laboratoire de Thermocinétique, CNRS UMR 6607, Ecole Polytechnique de l'Université de Nantes rue Christian Pauc, \\ 44306 Nantes Cedex 3, France
}

This paper deals with the transient motions experienced by an elastic circular cylinder in a cylindrical fluid domain initially at rest and subjected to small-amplitude imposed displacements. Three fluid models are considered, namely potential, viscous and acoustic, to cover different fluid-structure interaction regimes. They are derived here from the general compressible Navier-Stokes equations by a formal perturbation method so as to underline their links and ranges of validity a priori. The resulting fluid models are linear owing to the small-amplitude-displacement hypothesis. For simplicity, the elastic flexure beam model is chosen for the circular cylinder dynamics. The semi-analytical approach used here is based on the methods of Laplace transform in time, in vacuo eigenvector expansion with time-dependent coefficients for the transverse beam displacement and separation of variables for the fluid. Moreover, the viscous case is handled with a matched asymptotic expansion performed at first order. The projection of the fluid forces on the in vacuo eigenvectors leads to a fully coupled system involving the modal time-dependent displacement coefficients. These coefficients are then obtained by matrix inversion in the Laplace domain and fast numerical inversion of the Laplace transform. The three models, written in the form of convolution products, are described through the analysis of their kernels, involving both the wave propagation phenomena in the fluid domain and the beam elasticity. Last, the three models are illustrated for a specific imposed motion mimicking shock loading. It is shown that their combination permits coverage of a broad range of motions.

Keywords: Semi-analytical method; Asymptotic expansion; Laplace transform; Eigenvector expansion; Transient dynamics; Response function; Shock loading

\footnotetext{
${ }^{*}$ Corresponding author. Tel.: + 33240848784 .

E-mail address: jean-francois.sigrist@densgroup.com (J.F. Sigrist).

${ }^{1}$ Current address: LEPTIAB, Université de la Rochelle, Pôle Science et Technologie, Avenue Michel Crépeau, 17042 La Rochelle Cedex 1, France.
} 


\begin{tabular}{|c|c|c|c|}
\hline \multicolumn{2}{|c|}{ Nomenclature } & $t$ & time \\
\hline \multirow[t]{2}{*}{$C_{i}^{n}$} & $n$th fluid forces response to an impulsional & $\mathscr{W}$ & dimensionless frequency \\
\hline & motion of the whole system & $x$ & relative beam axis displacement in the $\mathbf{e}_{\mathbf{x}}-$ \\
\hline$C_{m}^{n}$ & $\begin{array}{l}n \text {th fluid forces response to an impulsional } \\
\text { motion of the } m \text { th beam mode }\end{array}$ & $x_{i}$ & $\begin{array}{l}\text { direction } \\
\text { imposed motion on the whole system }\end{array}$ \\
\hline $\begin{array}{l}c_{f} \\
E\end{array}$ & $\begin{array}{l}\text { speed of sound in the fluid at rest } \\
\text { elastic modulus }\end{array}$ & $x_{n}$ & $\begin{array}{l}n \text {th time-dependent modal displacement } \\
\text { coefficient }\end{array}$ \\
\hline$F$ & fluid loading in the $\mathbf{e}_{\mathbf{x}}$-direction & $z$ & vertical coordinate \\
\hline$f_{n}$ & $\begin{array}{l}n \text {th modal time-dependent fluid force } \\
\text { coefficient }\end{array}$ & $\begin{array}{l}\alpha \\
\beta\end{array}$ & $\begin{array}{l}\text { confinement ratio } \\
\text { Stokes number }\end{array}$ \\
\hline$H_{n}$ & $\begin{array}{l}\text { beam response function related to the } n \text {th } \\
\text { modal displacement coefficient }\end{array}$ & $\begin{array}{l}\gamma \\
\varepsilon\end{array}$ & $\begin{array}{l}\text { aspect ratio } \\
\text { perturbation parameter }\end{array}$ \\
\hline $\begin{array}{l}I \\
K\end{array}$ & $\begin{array}{l}\text { area moment of inertia } \\
\text { Keulegan-Carpenter number }\end{array}$ & $\lambda_{n}$ & $\begin{array}{l}n \text {th in vacuo eigenvalue of the beam axis } \\
\text { displacement }\end{array}$ \\
\hline$L$ & beam length & $v$ & kinematic fluid viscosity \\
\hline $\mathscr{M}$ & density ratio & $\rho$ & dynamic fluid density \\
\hline $\mathscr{M}_{a}$ & Mach number & $\rho_{s}$ & beam density \\
\hline$p$ & fluid pressure & $\rho_{f}$ & fluid density at rest \\
\hline$R_{1}$ & circular beam radius & $\dot{\phi}$ & velocity potential \\
\hline $\begin{array}{l}R_{2} \\
r\end{array}$ & $\begin{array}{l}\text { rigid outer circular cylinder radius } \\
\text { radial coordinate }\end{array}$ & $\varphi_{m}^{n}$ & $\begin{array}{l}\text { projection of the } n \text {th beam eigenfunction on } \\
\text { the } m \text { th vertical fluid mode }\end{array}$ \\
\hline$S$ & beam cross section & $\Omega$ & compressibility number \\
\hline$s$ & Laplace variable & $\omega_{n}$ & $n$th modal frequency \\
\hline
\end{tabular}

\section{Introduction}

When subjected to the effects of underwater explosions, naval propulsion devices can experience highly accelerated high-frequency motions (Keil, 1961; O'Hara and Cunnif, 1993). These components can be in contact with a fluid, as is the case for heat exchangers and nuclear propulsion reactors (Sigrist et al., 2006a, b; Sigrist and Broc, 2007). In order to improve design margins and ensure safety and satisfactory operating performance of the shock-loaded components, precise knowledge of their transient response is required. Since the body motion creates a fluid flow that in turn influences the body motion, complex fluid-structure interaction problems must be taken into account. In a first modelling step, design engineers must identify the meaningful physical phenomena in their particular geometry, such as viscous damping or compressibility effects. This can be achieved by studying simple representative fluid-structure problems for which analytical or semi-analytical solutions are available. The case of a circular cylinder confined in cylindrical fluid domains has already attracted a lot of attention due to its recurrence in the design of naval and nuclear components. However, the numerous studies available in (Gibert, 1986; Chen, 1987) focus on the harmonic dynamics. The transient case, which is of major importance in shock loading, has received comparatively less attention. Although the solution of a time-dependent problem can be formally obtained from the solution of the corresponding harmonic problem using Fourier synthesis methods (Landau and Lifshitz, 1959) or series of resonance modes (Habault and Filippi, 2003, 2004), time-domain methods are believed to provide more physical insight. In addition, these methods are currently receiving renewed interest (Stepanishen, 1997; Iakovlev, 2002, 2004, 2006, 2007), in particular due to increasing computer capabilities. The goal of the present paper is to formulate a time-domain method for a clampedfree elastic circular cylinder, confined in a cylindrical fluid domain initially at rest and subjected to a transient motion along a radial line; see Fig. 1. In addition to its industrial applications, this simple system is of academic concern since it illustrates in a closed form numerous fluid-structure interaction phenomena, such as structural mode coupling by the fluid, viscous damping, and some elasticity-compressibility interaction effects. The most restricting aspect of this study lies in the small-amplitude motion hypothesis. This is nevertheless reasonable for the design of numerous structures and helps lay the foundations for the understanding of more general fluid-structure interaction problems.

In order to cover a broad loading range, three fluid models are considered: potential, viscous and acoustic. Although their corresponding equations are classic (Lamb, 1932), their links and ranges of validity are not always explicit when used separately. In order to get some insight on their a priori limits, they are derived here from the compressible Navier-Stokes equations by a formal perturbation method. Owing to the small-amplitude-displacement hypothesis, the resulting fluid models are linear. The fluid forces, expressed for arbitrary motions of their boundaries and put into the 


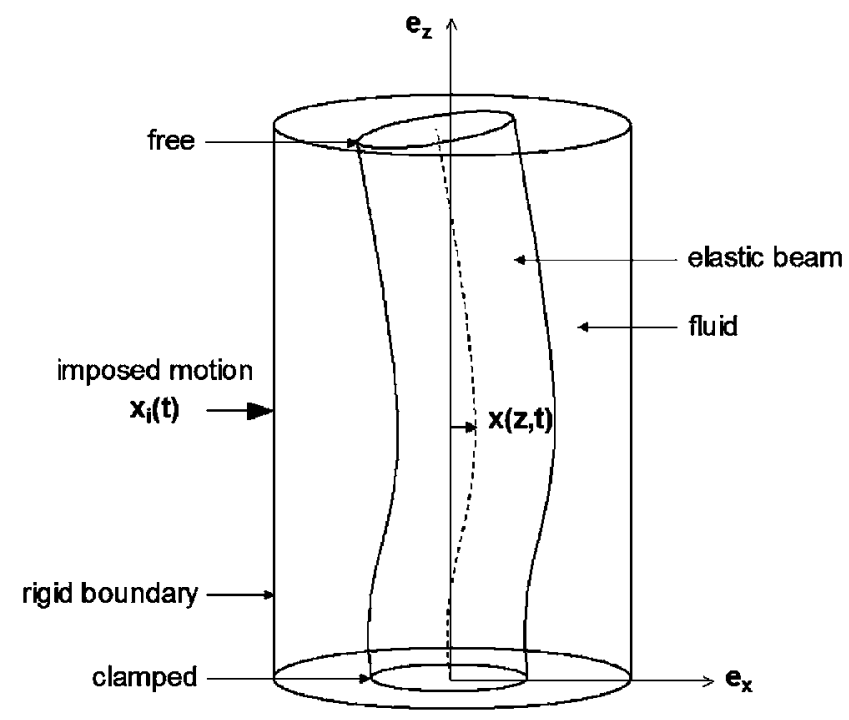

Fig. 1. Clamped-free beam in a cylindrically confined fluid domain.

form of convolution products, are derived by separation of variables. Moreover, for the viscous model, a first-order matched asymptotic expansion (Kevorkian and Cole, 1996), also called the composite boundary-layer theory (Schlichting, 1979), is performed. For simplicity, an elastic flexure beam is chosen to model the circular cylinder dynamics even though a more advanced model could be used with the same approach. Its displacement field is expressed by an in vacuo eigenvector expansion with time-dependent coefficients, which are the problem unknowns.

The right-hand side of the modal equations contains the projection of the fluid forces on the corresponding in vacuo eigenvector. The resulting coefficients for the modal fluid forces take the form of an infinite sum of temporal convolution integrals involving the time-dependent displacement coefficients. Finally, the problem is reduced to an infinite number of fully coupled integro-differential equations. The linearity of this time-dependent fluid-structure interaction problem suggests to employ the Laplace transform to turn it into a simple algebraic system. By truncating it to a finite number of modes, the displacement coefficients can be obtained by matrix inversion in the Laplace domain. A numerical inverse Laplace transform must then be performed to return to the temporal domain. Due to recent developments involving FFT-based and quotient-difference algorithms (Brancik, 2001, 2003), this operation is now a routine task and accurate Laplace transform inversions can be performed rapidly. Last, the transverse displacement field is found by modal recomposition.

This approach therefore combines analytical methods (separation of variables, regular and singular perturbation methods, Laplace transform, in vacuo eigenfunction decomposition) and numerical operations (matrix and Laplace transform inversions). This is a strong coupling procedure that provides converged fluid-structure solutions in a particular geometry. However, perturbation methods are used and the number of modes is truncated, so the solutions cannot be seen as exact. Nevertheless, their accuracy is limited mainly by the modelling and not by the numerical steps. The convergence analysis has been carried out for each case considered: keeping three to nine bending modes appears to be sufficient to obtain the series convergence, depending on the imposed solicitation and geometrical parameters. Furthermore, the solutions contribute to a physical understanding of the problem and can provide some elements traditionally difficult to evaluate by direct numerical simulations, such as the order of magnitude of the viscous damping in three-dimensional high-Stokes-number problems. It is thus hoped that these solutions be suitable for validating more evolved numerical codes, useful in solving fluid-structure interaction problems in complex geometries.

In the first part, the structural dynamics is introduced. The modal decomposition is recalled for the sake of completeness, although it could be found in classical textbooks (Blevins, 1984). In the second part, the fluid models are derived from the compressible Navier-Stokes equations by means of a formal regular perturbation method. Then the fluid-structure interaction problem is solved and described for each model. The effects of waves propagating in the fluid domain are stressed, such as the damping caused by the evanescent viscous shear waves and the reloading due to the acoustic pressure wave rebounds. Last, the solutions are illustrated for a specific imposed motion representative of a shock loading. It is shown that their combination permits coverage of a broad range of motions. 


\section{Beam dynamics}

We consider a clamped-free slender circular beam of radius $R_{1}$ and length $L$ confined in a cylindrical fluid domain limited by a rigid outer circular cylinder of radius $R_{2}$. A schematic of the problem is shown in Fig. 1 . The beam is assumed uniform along the span and composed of a linear, homogeneous, isotropic elastic material of density $\rho_{s}$. A transient motion $x_{i}(t)$ is imposed in the $\mathbf{e}_{\mathbf{x}}$-direction on the whole system so that the beam is put into motion through the clamped boundary condition and influence of the surrounding fluid. The imposed motion is assumed to be of sufficiently small amplitude that the induced transverse flexural beam displacement is negligible in relation to both its radius and the radial clearance. Neglecting shear deformations and considering only those normal to the undeformed beam axis gives the classical dynamics equation of flexure beams (Axisa, 2001). Normalizing the variables according to Table $1(\mathrm{a})$, where $U_{i}$ and $\omega_{i}$ are the characteristic velocity and frequency of the imposed motion and $\rho_{f}$ the fluid density, gives the following dimensionless equation with the clamped-free boundary conditions:

$$
\begin{aligned}
& \frac{\partial^{2} x}{\partial t^{2}}(z, t)+\mathscr{W}^{2} \frac{\partial^{4} x}{\partial z^{4}}(z, t)=-\ddot{x}_{i}(t)+\mathscr{M} F(z, t), \\
& \left.x\right|_{z=0}=0,\left.\quad \frac{\partial x}{\partial z}\right|_{z=0}=0,\left.\quad \frac{\partial^{2} x}{\partial z^{2}}\right|_{z=1}=0,\left.\quad \frac{\partial^{3} x}{\partial z^{3}}\right|_{z=1}=0,
\end{aligned}
$$

where $x(z, t)$ is the relative beam axis displacement, $z$ the vertical coordinate, $t$ the time and $F(z, t)$ the fluid loading in the $\mathbf{e}_{\mathbf{x}}$-direction. The two dimensionless numbers, $\mathscr{W}$ and $\mathscr{M}$, are defined by

$$
\mathscr{W}=\sqrt{\frac{E I}{\rho_{s} S L^{4} \omega_{i}^{2}}}, \quad \mathscr{M}=\frac{\rho_{f}}{\rho_{s}},
$$

with $S$ the cross-section, $E$ the elastic modulus and $I$ the area moment of inertia. $\mathscr{W}$ will be called the dimensionless frequency and quantifies the beam stiffness in relation to the imposed motion frequency. $\mathscr{M}$ is the density ratio. The beam axis displacement is sought in the form

$$
x(z, t)=\sum_{n=1}^{\infty} x_{n}(t) \varphi_{n}(z),
$$

Table 1

Normalization factors for the beam and fluid variables

Variable Normalization factor

(a) For the beam variables

Horizontal length

Vertical length

Time

Displacement

Acceleration

Fluid loading

(b) For the fluid variables

Length

Time

Displacement

Velocity

Pressure

Density
$R_{1}$
$L$
$1 / \omega_{i}$
$U_{i} / \omega_{i}$
$U_{i} \omega_{i}$
$\rho_{f} \pi R_{1}^{2} U_{i} \omega_{i}$

$R_{1}$

$1 / \omega_{i}$

$U_{i} / \omega_{i}$

$U_{i}$

$\rho_{f} c_{f}^{2}$

$\rho_{f}$ 
where $x_{n}(t)$ are the time-dependent modal displacement coefficients and the functions $\varphi_{n}(z)$ the in vacuo eigenfunctions of the beam axis displacement. These eigenfunctions can effectively be used without loss of generality for a fluid-structure interaction problem since they form a complete set; they are thus independent of the fluid model and can easily be derived by classical numerical methods. In vacuo and for the considered geometry, they satisfy the problem,

$$
\mathscr{W}^{2} \frac{\mathrm{d}^{4} \varphi_{n}}{\mathrm{~d} z^{4}}(z)=\omega_{n}^{2} \varphi_{n}(z),\left.\quad \varphi_{n}\right|_{z=0}=\left.\varphi_{n}^{\prime}\right|_{z=0}=\left.\varphi_{n}^{\prime \prime}\right|_{z=1}=\left.\varphi_{n}^{\prime \prime \prime}\right|_{z=1}=0
$$

where $\omega_{n}$ is the $n$th dimensionless modal frequency defined by $\omega_{n}=\mathscr{W} \lambda_{n}^{2}$ with $\lambda_{n}$ the $n$th eigenvalue. An analytical solution (Blevins, 1984) takes the form, for $n \geqslant 1$,

$$
\varphi_{n}(z)=\cosh \left(\lambda_{n} z\right)-\cos \left(\lambda_{n} z\right)+\frac{\cos \lambda_{n}+\cosh \lambda_{n}}{\sin \lambda_{n}+\sinh \lambda_{n}}\left[\sin \left(\lambda_{n} z\right)-\sinh \left(\lambda_{n} z\right)\right],
$$

where the first eigenvalues are given by $\lambda_{1}=1.8751, \lambda_{2}=4.6941, \lambda_{3}=7.8548, \lambda_{4}=10.9955, \lambda_{5}=14.1372$ and the following ones by $\lambda_{n}=\pi / 2+\pi(n-1)$ with good accuracy. The eigenfunctions satisfy the orthogonality relation,

$$
\int_{0}^{1} \varphi_{n}(z) \varphi_{m}(z) \mathrm{d} z=\delta_{n m}
$$

Substitution of the modal decomposition, Eq. (4), for the displacement in Eq. (1), followed by the use of Eq. (5), multiplication of the resulting relation by $\varphi_{m}(z)$, and integration along the beam length, yield the modal equation for the time-dependent displacement coefficient:

$$
\ddot{x}_{n}(t)+\omega_{n}^{2} x_{n}(t)=-\varphi_{n}^{0} \ddot{x}_{i}(t)+\mathscr{M} f_{n}(t), \quad f_{n}(t)=\int_{0}^{1} F(z, t) \varphi_{n}(z) \mathrm{d} z .
$$

The quantity $f_{n}(t)$ is the projection of the fluid forces on the $n$th eigenfunction and is called the modal fluid force coefficient. The coefficient $\varphi_{n}^{0}$ weights the inertial force and is a particular case of the following coefficients:

$$
\varphi_{m}^{q}=\int_{0}^{1} \varphi_{m}(z) \cos (q \pi z) \mathrm{d} z, \quad m \geqslant 1, q \geqslant 0 .
$$

In order to solve the modal Eq. (8) for all $n \geqslant 1$, the fluid forces must be expressed explicitly. This is the purpose of the following sections.

\section{Fluid dynamics}

\subsection{General equations}

The fluid medium is assumed continuous, homogeneous, Newtonian and without thermal effects. The mass and momentum conservation laws provide the compressible Navier-Stokes equations governing the fluid dynamics (Landau and Lifshitz, 1959). Normalizing the variables according to Table 1(b) with $c_{f}$ the isentropic sound velocity, and assuming a constant kinematic viscosity $v$, the dimensionless system takes the form:

$$
\begin{aligned}
& \frac{\partial \rho}{\partial t}+K \nabla \cdot(\rho \mathbf{u})=0, \\
& \frac{\partial(\rho \mathbf{u})}{\partial t}+K \nabla \cdot(\rho \mathbf{u} \otimes \mathbf{u})=-K \mathscr{M}_{a}^{-2} \nabla p+\beta^{-1} \nabla \cdot\left[\rho\left(\nabla \mathbf{u}+\nabla^{\mathrm{T}} \mathbf{u}-\frac{2}{3} \nabla \cdot \mathbf{u}\right)\right],
\end{aligned}
$$

where $\rho$ is the fluid density, $\mathbf{u}$ the velocity field and $p$ the pressure. The dimensionless numbers are defined in Eq. (16). The energy conservation law is not recalled since it is not useful for the simplified models derived in the following. In order to simplify the notation and without loss of generality, the vertical length and velocity are here normalized with the same quantities as the horizontal ones. They are renormalized by $L$ and $U_{i} R_{1} / L$, respectively, in Section 4 so as to be consistent with the notations used for the beam dynamics. The system of Eqs. (10) and (11) contains more unknowns than equations but can be closed by employing further hypotheses and introducing relations between the variables such as the dimensionless state equation,

$$
\mathrm{d} p=\mathrm{d} \rho+\left(\frac{\partial p}{\partial s}\right)_{\rho} \mathrm{d} s,
$$


with $s$ the entropy. The dimensionless no-slip boundary conditions on the moving boundaries take the form

$$
\left.\mathbf{u}\right|_{\mathscr{C}_{\text {int }}}=\left[\dot{x}(z, t)+\dot{x}_{i}(t)\right] \mathbf{e}_{\mathbf{x}}-\cos \theta \frac{\partial \dot{x}}{\partial z}(z, t) \mathbf{e}_{\mathbf{z}},\left.\quad \mathbf{u}\right|_{\mathscr{C}_{\text {ext }}}=\dot{x}_{i}(t) \mathbf{e}_{\mathbf{x}}
$$

where $\mathscr{C}_{\text {int }}$ and $\mathscr{C}_{\text {ext }}$ denote the surfaces of the inner and outer cylinders, see Fig. 1. The dimensionless position of the inner boundary is geometrically characterized by

$$
\mathbf{O M}=\left\{\cos \theta+K\left[x(z, t)+x_{i}(t)\right]\right\} \mathbf{e}_{\mathbf{x}}+\sin \theta \mathbf{e}_{\mathbf{y}}+\left[z-K \cos \theta \frac{\partial x}{\partial z}(z, t)\right] \mathbf{e}_{\mathbf{z}},
$$

with $O$ the point corresponding to the beam base and $M$ a point belonging to the inner surface $\mathscr{C}_{\text {int }}$. As for the other boundary, it is characterized by

$$
\mathbf{O M}=\left[\alpha \cos \theta+K x_{i}(t)\right] \mathbf{e}_{\mathbf{x}}+\alpha \sin \theta \mathbf{e}_{\mathbf{y}}+z \mathbf{e}_{\mathbf{z}}
$$

for a point $M$ belonging to the outer surface $\mathscr{C}_{\text {ext }}$. Moreover, the vertical fluid velocity is equal to zero at $z=0$ and $1 / \gamma$. Therefore, the system of Eqs. (10)-(15) is governed by the five dimensionless numbers:

$$
\alpha=\frac{R_{2}}{R_{1}}, \quad \gamma=\frac{R_{1}}{L}, K=\frac{U_{i}}{R_{1} \omega_{i}}, \beta=\frac{R_{1}^{2} \omega_{i}}{v}, \mathscr{M}_{a}=\frac{U_{i}}{c_{f}},
$$

where $\alpha$ and $\gamma$ are the confinement and aspect ratii and appear only in the boundary conditions; $K$, often called the Keulegan-Carpenter (1958) number, quantifies the cylinder displacements in relation to the beam radius and weights both the advection term in Eqs. (10) and (11) and the geometrical deformations in Eqs. (14) and (15). The Stokes number $\beta$ is the ratio between the unsteady and viscous effects, and the Mach number $\mathscr{M}_{a}$ is the ratio between the velocity imposed on the whole system and the speed of sound in the fluid at rest. All the models used in this article focus on small-Mach-number problems. For a steady flow, this condition is sufficient for neglecting compressible effects; however, for unsteady cases, it is not sufficient, and another dimensionless parameter must be introduced to characterize the influence of compressibility (Landau and Lifshitz, 1959). Anticipating Section 3.3, this number can be formed with the Mach and Keulegan-Carpenter numbers and is defined by

$$
\Omega=\frac{\mathscr{M}_{a}}{K}=\frac{R_{1} \omega_{i}}{c_{f}} .
$$

Several approximations and the asymptotic behaviour of the system of Eqs. (10)-(15) are considered in the following. In order to appreciate the underlying hypotheses, the simple models are obtained from a formal perturbation method applied to the general system. Since small-Mach-number flows are studied here and the Mach number appears only with the pressure-gradient term, a regular expansion involving the powers of $\mathscr{M}_{a}$ seems reasonable. Further analysis suggests taking as perturbation parameter $\varepsilon$ the quantity,

$$
\varepsilon=K^{-1} \mathscr{M}_{a}^{2} .
$$

Thus the dimensionless fluid variables are sought in the form

$$
\left\{\begin{array}{l}
\rho \\
\mathbf{u} \\
p
\end{array}\right\}=\left\{\begin{array}{l}
\rho_{0} \\
\mathbf{u}_{\mathbf{0}} \\
p_{0}
\end{array}\right\}+\varepsilon\left\{\begin{array}{l}
\rho_{1} \\
\mathbf{u}_{\mathbf{1}} \\
p_{1}
\end{array}\right\}+\mathscr{O}\left(\varepsilon^{2}\right) .
$$

The substitution of this expansion for the fluid variables into the dimensionless compressible Navier-Stokes equations together with different orders of magnitude for the parameters $K$ and $\beta$ allow us to obtain the simplified models.

\subsection{Incompressible behaviour: potential and viscous models}

\subsubsection{General incompressible limit}

In a first step, no assumption is made about the Stokes number. The Keulegan-Carpenter number is taken such that the perturbation parameter $\varepsilon$, defined by Eq. (18), tends to zero as the Mach number tends to zero, hence $\mathscr{M}_{a}^{2} \ll K$. The regular expansion (19) is substituted into the system of Eqs. (10) and (11). Assuming that $\rho_{0}$ is constant in space and time (so that its dimensional value is the fluid density at rest $\rho_{f}$ and $\rho_{0}=1$ ), the leading-order equations can be written as

$$
\nabla \cdot \mathbf{u}_{0}=0 \quad \text { and } \quad \nabla p_{0}=0 .
$$


Therefore, the leading-order pressure $p_{0}$ is constant in space and does not contribute to the fluid forces. The first-order equation is the classical incompressible Navier-Stokes equation, in a dimensionless form particularly adapted to the case of interest here, a fluid flow induced by moving boundaries:

$$
\frac{\partial \mathbf{u}_{0}}{\partial t}+K\left(\mathbf{u}_{0} \cdot \nabla\right) \mathbf{u}_{0}=-\nabla p_{1}+\beta^{-1} \nabla^{2} \mathbf{u}_{\mathbf{0}} .
$$

Hence the first-order pressure $p_{1}$ is not constant and interacts with the leading-order velocity field. This differs from flows that are not induced by moving boundaries, for instance Poiseuille flow, for which even the first-order pressure is null and only the second-order pressure interacts with the leading-order velocity field (Schmid and Henningson, 2001). By bringing together the normalization factor for the pressure term in Table 1(b) with expansion (19), the effective normalization factor for the pressure is found to be $\rho_{f} R_{1} U_{i} \omega_{i}$, which explains the fluid loading factor used in Table 1(a). In shock loading, the internal structures are mainly subjected to small-amplitude, high-frequency motions. Hence the Keulegan-Carpenter number is small (which implies here $\mathscr{M}_{a}^{2} \ll K \ll 1$ ) and both the advection term in the Navier-Stokes Eq. (21) and the geometrical deformations in Eqs. (14) and (15) can be disregarded. The resulting governing equations are given by

$$
\nabla \cdot \mathbf{u}_{\mathbf{0}}=0, \quad \frac{\partial \mathbf{u}_{0}}{\partial t}=-\nabla p_{1}+\beta^{-1} \nabla^{2} \mathbf{u}_{0}
$$

with the no-slip boundary conditions, now expressed at fixed positions,

$$
\left.\mathbf{u}_{\mathbf{0}}\right|_{r=1}=\left[\dot{x}(z, t)+\dot{x}_{i}(t)\right] \mathbf{e}_{\mathbf{x}}-\cos \theta \frac{\partial \dot{x}}{\partial z}(z, t) \mathbf{e}_{\mathbf{z}},\left.\quad \mathbf{u}_{\mathbf{0}}\right|_{r=\alpha}=\dot{x}_{i}(t) \mathbf{e}_{\mathbf{x}} .
$$

This system of equations is the starting point of both the potential and the viscous boundary-layer models.

\subsubsection{Potential model}

The potential model is derived from the system of Eqs. (22) and (23) by taking the limit of an infinite Stokes number $(\beta \rightarrow \infty)$. Since the second-order space derivative is lost in Eq. (22), all the boundary conditions cannot be satisfied. Physical arguments suggest conserving those normal to the boundaries, resulting in slip boundary conditions. Assuming that the flow is initially irrotational, it remains irrotational at later times (Lamb, 1932) and a velocity potential $\phi$ can be introduced so that its leading order satisfies $\mathbf{u}_{0}=\nabla \phi_{0}$. Then, a straightforward derivation shows that the flow is governed by the Laplace equation for the velocity potential,

$$
\nabla^{2} \phi_{0}=0
$$

with the Neumann-type boundary conditions

$$
\left.\frac{\partial \phi_{0}}{\partial r}\right|_{r=1}=\left[\dot{x}(z, t)+\dot{x}_{i}(t)\right] \cos \theta,\left.\quad \frac{\partial \phi_{0}}{\partial r}\right|_{r=\alpha}=\dot{x}_{i}(t) \cos \theta,\left.\frac{\partial \phi_{0}}{\partial z}\right|_{z=0,1 / \gamma}=0 .
$$

Once the system of Eqs. (24) and (25) is solved, the first-order fluid pressure can be found from the simplified Bernoulli equation,

$$
\frac{\partial \phi_{0}}{\partial t}+p_{1}=c(t)
$$

where $c(t)$ is constant in space and time-dependent. Since only the gradients of $\phi_{0}$ contribute to the integrated fluid forces for a fixed $z, c(t)$ can be absorbed without loss of generality in the velocity potential definition and thus taken as null in the above equation.

\subsubsection{Viscous boundary-layer model}

The beam displacement is assumed sufficiently small that no boundary-layer separation occurs (Schlichting, 1979). Hence, there is neither wake formation nor its force-related modifications (Sarpkaya, 1986; Koumoutsakos and Leonard, 1986). The fluid damping on a rigid oscillating cylinder in an infinite fluid domain was derived exactly by Stokes (1851) assuming laminar two-dimensional flow and neglecting the advection term. Approximate solutions have been derived by Stuart (1963) and Wang (1968), among others, in order to identify higher-order phenomena. These studies showed that a first-order boundary-layer theory is sufficient to characterize the viscous damping for highStokes-number laminar two-dimensional flows. However, it is now known that the attached boundary-layer, i.e. without separation, is subjected to three-dimensional centrifugal and transient instabilities (Honji, 1981; Hall, 1984; Sarpkaya, 2002). Their influence is manifested by the increase of the viscous damping by a factor of two over the Stokes 
theory (Sarpkaya, 2001), which does not significantly change its order of magnitude. Neither experimental nor theoretical stability analysis has been reported for confined fluid flow. Here, a matched asymptotic expansion of the first order is performed to build a laminar viscous boundary-layer on the transiently moving elastic beam in a confined fluid domain. By analogy with an infinite fluid domain and for high-Stokes-number flow, it is expected to give the order of magnitude of the viscous damping or at least a lower bound, even though it does not take into account any instabilities. The resulting fluid model is thought useful for cases where the viscous boundary layer is much smaller than the radial clearance, i.e. $\sqrt{v / \omega_{i}} \ll R_{2}-R_{1}$, or in dimensionless form, $\beta^{1 / 2}(\alpha-1) \gg 1$. This condition is more restricting than that in first-order boundary-layer theory for an infinite fluid domain given by $\beta^{1 / 2} \gg 1$.

The beam motion is assumed of sufficiently low amplitude and high frequency that the fluid domain can be divided into two parts, one far from the boundaries, where viscous effects can be neglected, and the other closer to the boundaries, where the viscous dissipation is a leading-order term (Kevorkian and Cole, 1996). Hence, far from the boundaries, the following outer expansion in powers of $\beta^{-1 / 2}$ is introduced into the nondimensional Eqs. (22) and (23),

$$
\left\{\begin{array}{l}
\mathbf{u}_{\mathbf{0}} \\
p_{1}
\end{array}\right\}=\left\{\begin{array}{l}
\mathbf{u}_{\mathbf{0}}^{0} \\
p_{1}^{0}
\end{array}\right\}+\beta^{-1 / 2}\left\{\begin{array}{l}
\mathbf{u}_{\mathbf{0}}^{1} \\
p_{1}^{1}
\end{array}\right\}+\mathcal{O}\left(\beta^{-1}\right) .
$$

The leading-order equations are given by

$$
\nabla \cdot \mathbf{u}_{\mathbf{0}}^{\mathbf{0}}=0, \quad \frac{\partial \mathbf{u}_{\mathbf{0}}^{\mathbf{0}}}{\partial t}=-\nabla p_{1}^{0}
$$

Assuming that $\mathbf{u}_{\mathbf{0}}^{\mathbf{0}}$ is initially irrotational, a velocity potential $\phi_{0}^{0}$ can be introduced such that $\mathbf{u}_{\mathbf{0}}^{\mathbf{0}}=\nabla \phi_{0}^{0}$. Therefore, $\phi_{0}^{0}$ satisfies the same system of equations as $\phi_{0}$ in the potential model, i.e., Eqs. (24) and (25). Since we are interested in the beam displacement, the boundary-layer is created only near its surface, not near that of the outer cylinder. In effect, at this order the outer cylinder viscous boundary-layer for a high-Stokes-number flow is not expected to go through the potential flow separating the two surfaces. Hence the outer boundary is taken into account only in its effect on the potential flow. Higher-order phenomena such as steady streaming (Riley, 2001) must include more complex interactions among the two boundaries, the potential flow and the advection term.

Close to the beam, the radial boundary layer variable $\eta=\beta^{1 / 2}(r-1)$ is introduced in the system of Eqs. (22) and (23). Furthermore the following inner expansion is performed:

$$
\left\{\begin{array}{c}
\bar{u}_{\eta} \\
\bar{u}_{\theta} \\
\bar{u}_{z} \\
\bar{p}
\end{array}\right\}=\left\{\begin{array}{c}
0 \\
\bar{u}_{\theta}^{0} \\
\bar{u}_{z}^{0} \\
\bar{p}^{0}
\end{array}\right\}+\beta^{-1 / 2}\left\{\begin{array}{c}
\bar{u}_{\eta}^{1} \\
\bar{u}_{\theta}^{1} \\
\bar{u}_{z}^{1} \\
\bar{p}^{1}
\end{array}\right\}+\mathcal{O}\left(\beta^{-1}\right),
$$

where $\bar{u}_{\eta}, \bar{u}_{\theta}, \bar{u}_{z}$ and $\bar{p}$ denote, respectively, the radial, azimuthal and vertical velocities and the fluid pressure inside the boundary-layer and depend on $\eta, \theta, z$, and $t$. The introduction of the boundary-layer variable $\eta$ together with the inner expansion Eq. (29) take into account the different orders of magnitude of the radial and azimuthal boundary-layer velocities: the radial velocity is normalized by the viscous diffusion velocity $\sqrt{v \omega_{i}}$ and the azimuthal velocity by the velocity imposed on the whole system. Thus the leading-order equations are given by

$$
\frac{\partial \bar{p}^{0}}{\partial \eta}=0 \quad \text { and } \quad \frac{\partial \bar{u}_{\theta}^{0}}{\partial t}=-\frac{\partial \bar{p}^{0}}{\partial \theta}+\frac{\partial^{2} \bar{u}_{\theta}^{0}}{\partial \eta^{2}} .
$$

Since evaluation of the fluid forces at a fixed $z$ requires only knowledge of the azimuthal component, $\bar{u}_{\theta}^{0}$, the equations governing the vertical and axial velocity components, $\bar{u}_{z}^{0}$ and $\bar{u}_{\eta}^{1}$, are not expressed here. The first relation of Eq. (30) states that the leading-order boundary-layer pressure is constant across the viscous boundary-layer. Hence it can be linked to the outer variables, typically by considering the limit, as $r$ tends to 1, of the azimuthal component of Eq. (28). Substituting the resulting relation in Eq. (30) yields the boundary-layer equation:

$$
\left(\frac{\partial}{\partial t}-\frac{\partial^{2}}{\partial \eta^{2}}\right) \bar{u}_{\theta}^{0}=\left.\frac{\partial u_{\theta, 0}^{0}}{\partial t}\right|_{r=1},
$$

where $u_{\theta, 0}^{0}$ is the azimuthal component of $\mathbf{u}_{\mathbf{0}}^{\mathbf{0}}$. The boundary layer velocity $\bar{u}_{\theta}^{0}$ must satisfy the no-slip boundary conditions on the beam surface, Eq. (23), and following the limit-matching principle (Van Dyke, 1964), must match as $\eta \rightarrow \infty$, the outer azimuthal velocity evaluated on the boundary, i.e., at $r=1$ :

$$
\left.\bar{u}_{\theta}^{0}\right|_{\eta=0}=-\left[\dot{x}(z, t)+\dot{x}_{i}(t)\right] \sin \theta \quad \text { and } \quad \lim _{\eta \rightarrow \infty} \bar{u}_{\theta}^{0}(\eta, \theta, z, t)=\left.u_{\theta, 0}^{0}\right|_{r=1} .
$$


Therefore, the boundary-layer azimuthal velocity can be derived using Eq. (31) together with the boundary conditions, Eq. (32), once the outer potential problem has been solved.

\subsection{Compressible behaviour: acoustic model}

Here modelling of fluid compressibility is investigated. This section differs from Section 3.2 in the order of magnitude used for the Keulegan-Carpenter and Stokes numbers:

$$
K=\mathcal{O}\left(\mathscr{M}_{a}\right) \ll 1 \text { and } \beta \rightarrow \infty .
$$

Hence the parameter $\varepsilon$ defined by Eq. (18) behaves as $\mathcal{O}\left(\mathscr{M}_{a}\right)$. Since small-Mach-number flows are considered, $\varepsilon$ is expected to be small enough to be used as a perturbation parameter and the expansion given by Eq. (19) is substituted into the system of dimensionless (10) and (11). Retaining terms of the same order and assuming that leading-order density $\rho_{0}$ is initially constant in space yield the following equations:

$$
\frac{\partial \rho_{1}}{\partial t}+\frac{K}{\varepsilon} \nabla \cdot \mathbf{u}_{0}=0 \quad \text { and } \quad \frac{\partial \mathbf{u}_{0}}{\partial t}=-\nabla p_{1} .
$$

A relation between $\rho_{1}$ and $p_{1}$ is required to close this system. Since the viscous dissipation is neglected at this order and thermal effects are not considered, the flow is isentropic. Hence, substituting the expansion, Eq. (19), in the dimensionless state Eq. (12) gives $p_{1}=\rho_{1}$. It is convenient to introduce the velocity potential $\phi$ so that its leading order satisfies $\mathbf{u}_{0}=\nabla \phi_{0}$. Its substitution into the second relation of Eq. (34) and integration according to the space variables give a Bernoulli relation identical to that obtained in the potential model, Eq. (26). Then, its substitution into the first relation of Eq. (34) provides the wave equation for the velocity potential,

$$
\nabla^{2} \phi_{0}-\Omega^{2} \frac{\partial^{2} \phi_{0}}{\partial t^{2}}=0 \quad \text { with } \Omega=\sqrt{\frac{\varepsilon}{K}}=\frac{R_{1} \omega_{i}}{c_{f}} .
$$

The compressibility number $\Omega$ appears to be the meaningful dimensionless parameter to quantify fluid compressibility effects. The Neumann-type boundary conditions are given by Eq. (25).

\section{Fluid-structure interaction}

\subsection{Resolution method}

The potential, viscous boundary layer and acoustic models discussed in Section 3 are linear, owing to the hypothesis of small beam displacements in relation to both the beam radius and the radial clearance. Furthermore, the modal Eqs. (8) are expected to be coupled by the fluid presence. These remarks suggest that the modal time-dependent fluid force coefficients $f_{n}(t)$, can be formulated as

$$
f_{n}(t)=\int_{0}^{t} C_{i}^{n}(t-\tau) \ddot{x}_{i}(\tau) \mathrm{d} \tau-\sum_{m=1}^{\infty} \int_{0}^{t} C_{m}^{n}(t-\tau) \ddot{x}_{m}(\tau) \mathrm{d} \tau .
$$

The function $C_{i}^{n}(t)$ denotes the fluid influence on the $n$th flexural beam mode, induced by an impulsional imposed motion and without relative motions, i.e. for $\ddot{x}_{i}(t)=\delta(t)$ and $\ddot{x}(z, t)=0$. The function $C_{m}^{n}(t)$ is the fluid influence on the $n$th mode induced by an impulsional motion of the $m$ th mode, i.e., for $\ddot{x}_{i}(t)=0$ and $\ddot{x}(z, t)=\delta(t) \varphi_{m}(z)$. The convolution products reflect that the fluid response is not instantaneous, except in the potential model, since wave-propagation phenomena in the fluid domain are involved. The fluid response functions $C_{i}^{n}(t)$ and $C_{m}^{n}(t)$ and their physical interpretation are described for each fluid model in the following sections. Once the modal fluid force coefficients are put into the form of Eq. (36), the fluid-structure interaction problem can be solved by the same approach for the three fluid models. The substitution of Eq. (36) into the modal Eq. (8) yields an infinite number of fully coupled integrodifferential equations. By truncating to a finite number of modes, the resulting system could be solved by a timemarching scheme. However, its linearity suggests the Laplace transform use to turn it into a simple algebraic system. Thus, employing the convolution theorem and introducing the complex functions $\hat{\chi}_{m}^{n}(s)$ and $\hat{\chi}_{i}^{n}(s)$ as

$$
\hat{\chi}_{m}^{n}(s)=\frac{s^{2} \mathscr{M} \hat{C}_{m}^{n}(s)}{s^{2}\left[1+\mathscr{M} \hat{C}_{n}^{n}(s)\right]+\omega_{n}^{2}}, \quad \hat{\chi}_{i}^{n}(s)=\frac{\mathscr{M} \hat{C}_{i}^{n}(s)-\varphi_{n}^{0}}{s^{2}\left[1+\mathscr{M} \hat{C}_{n}^{n}(s)\right]+\omega_{n}^{2}},
$$


each modal equation can be written in the form,

$$
\hat{x}_{n}(s)+\sum_{m=1, m \neq n}^{\infty} \hat{\chi}_{m}^{n}(s) \hat{x}_{m}(s)=\hat{\chi}_{i}^{n}(s) \hat{\ddot{x}}_{i}(s),
$$

where $s$ is the complex Laplace variable. Without fluid, i.e., for $\mathscr{M}=0$, the modes are uncoupled and Eq. (38) reduces to $\hat{x}_{n}(s)=-\hat{\ddot{x}}_{i}(s) \varphi_{n}^{0} /\left(s^{2}+\omega_{n}^{2}\right)$. By using some Laplace transform formulas, its solution in the temporal domain is simply given by $x_{n}(t)=-\varphi_{n}^{0} / \omega_{n} \int_{0}^{t} \sin \left[\omega_{n}(t-\tau)\right] \ddot{x}_{i}(\tau) \mathrm{d} \tau$. The system memory, reflected by the kernel $\sin \left(\omega_{n} t\right)$, is due only to the $n$th beam mode elasticity and illustrates the nondissipative character of the system. The functions $\hat{\chi}_{m}^{n}(s)$ and $\hat{\chi}_{i}^{n}(s)$ quantify, in the complex plane, respectively, the $m$ th mode influence on the $n$th mode dynamics and the way in which the imposed motion $\ddot{x}_{i}(t)$ is felt by the $n$th mode. Keeping only the first $N$ modal Eq. (38) lets us write the resulting truncated system in the matrix form

$$
\left[\begin{array}{ccccc}
1 & \hat{\chi}_{1}^{2}(s) & \cdots & \cdots & \hat{\chi}_{1}^{N}(s) \\
\hat{\chi}_{2}^{1}(s) & 1 & \ddots & & \vdots \\
\vdots & \ddots & \ddots & \ddots & \vdots \\
\vdots & & \ddots & 1 & \hat{\chi}_{N-1}^{N}(s) \\
\hat{\chi}_{N}^{1}(s) & \cdots & \cdots & \hat{\chi}_{N}^{N-1}(s) & 1
\end{array}\right]\left\{\begin{array}{c}
\hat{x}_{1}(s) \\
\hat{x}_{2}(s) \\
\vdots \\
\hat{x}_{N-1}(s) \\
\hat{x}_{N}(s)
\end{array}\right\}=\hat{\ddot{x}}_{i}(s)\left\{\begin{array}{c}
\hat{\chi}_{i}^{1}(s) \\
\hat{\chi}_{i}^{2}(s) \\
\vdots \\
\hat{\chi}_{i}^{N-1}(s) \\
\hat{\chi}_{i}^{N}(s)
\end{array}\right\}
$$

By denoting $[\hat{M}(s)]$ and $\{\hat{x}(s)\}$, the matrix and vector of the left-hand side, respectively, and $\left\{\hat{\chi}_{i}(s)\right\}$, the right-hand side vector, the first $N$ displacement coefficients can be found by matrix inversion, with the Laplace variable $s$ as a parameter, and by performing an inverse Laplace transform to return to the temporal domain:

$$
\{x(t)\}=\mathscr{L}^{-1}\left(\hat{\ddot{x}}_{i}(s)[\hat{M}(s)]^{-1}\left\{\hat{\chi}_{i}(s)\right\}\right)(t),
$$

where $\mathscr{L}^{-1}().(t)$ denotes the Laplace transform inversion. This operation is carried out by a FFT-based algorithm together with a quotient-difference algorithm to accelerate the FFT series convergence and thus enhance solution accuracy (Brancik, 2001, 2003). If we denote by $\{\varphi(z)\}$ the vector containing the first $N$ bending modes, the displacement field, $x(z, t)$, is deduced from the modal decomposition as

$$
x(z, t)=\langle x(t)\rangle\{\varphi(z)\} .
$$

For further physical interpretation, it can be fruitful to use the convolution theorem during the Laplace transform inversion. Moreover, by denoting $\hat{H}_{n}(s)$ the $n$th component of the vector $[\hat{M}(s)]^{-1}\left\{\hat{\chi}_{i}(s)\right\}$ and $H_{n}(t)$ its corresponding temporal function, the $n$th modal displacement coefficient $x_{n}(t)$, and the displacement field $x(z, t)$ can be written as

$$
x_{n}(t)=\int_{0}^{t} H_{n}(t-\tau) \ddot{x}_{i}(\tau) \mathrm{d} \tau, \quad x(z, t)=\sum_{n=1}^{N} \varphi_{n}(z) \int_{0}^{t} H_{n}(t-\tau) \ddot{x}_{i}(\tau) \mathrm{d} \tau .
$$

The kernel $H_{n}(t)$ is the response function of the $n$th modal displacement coefficient. It involves the elasticity of the $n$th mode and the influence of the $N-1$ others as a result of the fluid coupling. It also reflects the influence of the wave propagation phenomena in the fluid domain. Since it is independent of the imposed motion, some characteristics of the fluid-structure interaction problem can be assessed from a general viewpoint. The fluid response functions $C_{i}^{n}(t)$ and $C_{m}^{n}(t)$ and the beam response functions $H_{n}(t)$ are still to be expressed explicitly. They are derived and described for each fluid model in what follows.

\subsection{Interaction with potential flow}

Assuming that $\beta \rightarrow \infty, \mathscr{M}_{a}^{2} \ll K \ll 1$ and $\Omega \ll 1$, the fluid dynamics is governed by the Laplace Eq. (24) for the velocity potential with the Neumann-type boundary conditions, Eq. (25). The vertical length and velocity are now renormalized, respectively, by $L$ and $U_{i} R_{1} / L$ instead of $R_{1}$ and $U_{i}$ so as to be consistent with the notations used for the beam dynamics. Expressing the Laplace equation in a cylindrical coordinate system, using separation of variables and Eqs. (4) and (25) yield the velocity potential

$$
\phi_{0}(r, \theta, z, t)=r \dot{x}_{i}(t) \cos \theta-\sum_{m=1}^{\infty}\left[\sum_{q=0}^{\infty} \varphi_{m}^{q} c_{q}(r) \cos (q \pi z)\right] \dot{x}_{m}(t) \cos \theta,
$$


where the functions $c_{q}(r)$ are given by

$$
\begin{aligned}
& c_{0}(r)=\frac{1}{\alpha^{2}-1}\left(\frac{\alpha^{2}}{r}+r\right), \\
& c_{q}(r)=-\frac{2}{k_{q}} \frac{\mathrm{Y}_{1}^{\prime}\left(k_{q} \alpha\right) \mathrm{J}_{1}\left(k_{q} r\right)-\mathrm{J}_{1}^{\prime}\left(k_{q} \alpha\right) \mathrm{Y}_{1}\left(k_{q} r\right)}{\mathrm{J}_{1}^{\prime}\left(k_{q}\right) \mathrm{Y}_{1}^{\prime}\left(k_{q} \alpha\right)-\mathrm{J}_{1}^{\prime}\left(k_{q} \alpha\right) \mathrm{Y}_{1}^{\prime}\left(k_{q}\right)} \quad \text { for } q \geqslant 1,
\end{aligned}
$$

with $J_{1}$ and $Y_{1}$ the Bessel functions of order 1 of the first and second kind, respectively (Abramowitz and Stegun, 1970), $k_{q}$ defined by $k_{q}=\mathrm{i} q \pi \gamma$ and $\varphi_{m}^{q}$ given by Eq. (9). In the potential flow model, the dimensionless integrated fluid forces at a fixed position $z$ are due to the fluid pressure alone. At this order, only its $\mathbf{e}_{\mathbf{x}}$-direction component is nonzero. The firstorder fluid pressure $p_{1}$ can be deduced from the velocity potential Eqs. (43)-(45) and the Bernoulli relation, Eq. (26). Its evaluation on the beam surface and integration according to $\theta$ yield the expression for the fluid forces. Then, their projections on the $n$th eigenfunction give the modal time-dependent coefficients

$$
f_{n}(t)=\varphi_{n}^{0} \ddot{x}_{i}(t)-\sum_{m=1}^{\infty}\left[\sum_{q=0}^{\infty} \varphi_{m}^{q} \varphi_{n}^{q} c_{q}\right] \ddot{x}_{m}(t),
$$

where $c_{q}=c_{q}(1)$. The first term on the right-hand side weights the inertial effect. The second involves all the beam modes; it is proportional, for each $m$, to the modal acceleration $\ddot{x}_{m}(t)$. Hence the coefficient $\sum_{q} \varphi_{m}^{q} \varphi_{n}^{q} c_{q}$ can be seen as the added mass induced by the $m$ th mode motion and transmitted on the $n$th mode by the fluid. The modal fluid force coefficients can also be put into convolution-product form, Eq. (36), with the kernels,

$$
C_{i}^{n}(t)=\varphi_{n}^{0} \delta(t) \quad \text { and } \quad C_{m}^{n}(t)=\sum_{q=0}^{\infty} \varphi_{m}^{q} \varphi_{n}^{q} c_{q} \delta(t) .
$$

The Dirac distribution $\delta(t)$ reflects the obvious result that a potential flow responds instantaneously and everywhere to the beam motion, without history effect. The function $C_{m}^{n}(t)$, which is the fluid influence on the $n$th mode induced by an impulsional motion of the $m$ th mode, contains the contribution of all the vertical fluid modes. The beam response functions, $H_{n}(t)$, can now be described. They are illustrated in Fig. 2(a) for $n=1$ with dimensionless parameters $\mathscr{W}=1, \mathscr{M}=\frac{1}{8}, \alpha=2$, and $\gamma=0.2$. The dotted lines correspond to the response functions obtained without fluid and simply given by $-\varphi_{n}^{0} / \omega_{n} \sin \left(\omega_{n} t\right)$. The major effect of the fluid is to reduce for each mode both the displacement amplitude and frequency. Although $H_{1}(t)$ involves the elasticity of all the bending modes due to the fluid coupling, that of the first mode is strongly dominant.

\subsection{Interaction with viscous flow}

Assuming that $\beta^{1 / 2}(\alpha-1) \gg 1, \mathscr{M}_{a}^{2} \ll K \ll 1$ and $\Omega \ll 1$, a viscous boundary-layer is formed near the beam surface. To solve the boundary-layer Eqs. (31) and (32), the azimuthal velocity of the outer flow, $u_{\theta, 0}^{0}$, is needed. Since the flow far

(a)

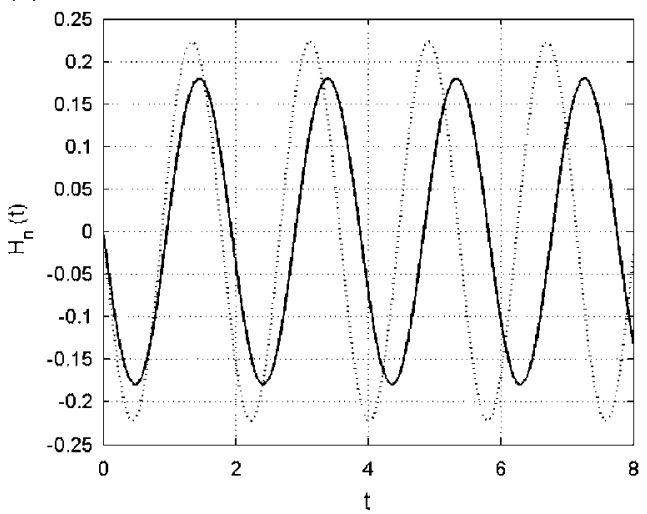

(b)

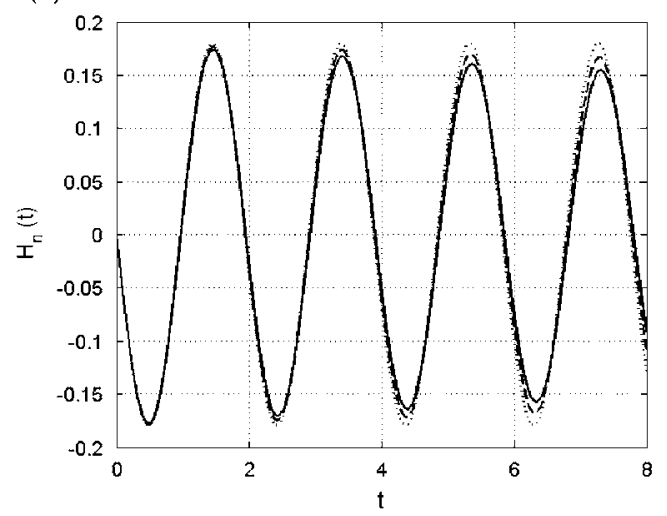

Fig. 2. The first response function $H_{1}(t)$ for $\mathscr{W}=1, \mathscr{M}=\frac{1}{8}, \alpha=2$ and $\gamma=0.2$. (a) -, potential; $\cdots$, in vacuo and (b) $\cdots$, potential; viscous with,$-- \beta=1000 ;-, \beta=250$. 
from the boundaries is potential at the leading order, the velocity potential $\phi_{0}^{0}$ is equal to that obtained in Eqs. (43)-(45) and $u_{\theta, 0}^{0}$ can then be deduced,

$$
u_{\theta, 0}^{0}(r, \theta, z, t)=-\left\{\dot{x}_{i}(t)-\frac{1}{r} \sum_{m=1}^{\infty}\left[\sum_{q=0}^{\infty} \varphi_{m}^{q} c_{q}(r) \cos (q \pi z)\right] \dot{x}_{m}(t)\right\} \sin \theta .
$$

By expressing the boundary-layer Eqs. (31) and (32) in the Laplace domain and by using the above expression, the boundary-layer solution is obtained with separation of variables:

$$
\frac{\hat{\bar{u}}_{\theta}^{0}(\eta, \theta, z, s)}{\sin \theta}=-\hat{\dot{x}}_{i}(s)+\sum_{m=1}^{\infty}\left[\left(1-\mathrm{e}^{-\eta \sqrt{s}}\right) \sum_{q=0}^{\infty} \varphi_{m}^{q} c_{q} \cos (q \pi z)-\mathrm{e}^{-\eta \sqrt{s}} \varphi_{m}(z)\right] \hat{\dot{x}}_{m}(s) .
$$

The solution, uniformly valid in the fluid domain and denoted by $\hat{u}_{\theta}$, is derived by summing the above relation with the outer one given in Eq. (48) and removing the common limit, which is counted twice (Kevorkian and Cole, 1996). Moreover, performing the change of variable $\eta=\beta^{1 / 2}(r-1)$ and employing the usual inverse Laplace transform relations, let us express the solution in the form

$$
u_{\theta}(r, \theta, z, t)=-\sin \theta \dot{x}_{i}(t)+\sum_{m=1}^{\infty} \int_{0}^{t} Q_{m}(r, \theta, z, t-\tau) \ddot{x}_{m}(\tau) \mathrm{d} \tau,
$$

where the function $Q_{m}(r, \theta, z, t)$ is the fluid velocity response to an impulsional motion of the $m$ th flexure beam mode, defined by

$$
Q_{m}(r, \theta, z, t)=u(t) \sum_{q=0}^{\infty} \varphi_{m}^{q} c_{q}(r) \cos (q \pi z) \sin \theta-\operatorname{erfc}\left(\frac{r-1}{2} \sqrt{\frac{\beta}{t}}\right)\left[\varphi_{m}(z)+\sum_{q=0}^{\infty} \varphi_{m}^{q} c_{q} \cos (q \pi z)\right] \sin \theta,
$$

with $u(t)$ and $\operatorname{erfc}(t)$ the Heaviside and complementary error function (Abramowitz and Stegun, 1970), respectively. The first term on the right-hand side describes the potential flow contribution, while the second is due only to the first-order viscous correction and vanishes as $r$ increases. The fluid velocity response induced by the impulsional motion of the third flexure beam mode, $Q_{3}$, is illustrated in Fig. 3. The fields are displayed in the $(r, z)$ plane at $\theta=\pi / 2$, for half the radial clearance and with dimensionless parameters $\alpha=2, \gamma=0.2$, and $\beta=500$. The dotted lines in Fig. 3(b) and (c) denote the positions in the $(r, z)$ plane at which the relative error between the viscous azimuthal velocity and that obtained with the potential model is $1 \%$. An evanescent shear wave generated on the beam surface at $t=0$ is seen to propagate in the fluid domain as $t$ increases. In parallel, the shear at the boundary, initially infinite, decreases as the wave propagates. Obviously, the viscous velocity field matches the potential flow for large radial positions $r$. This effect occurs more quickly as the Stokes number is large and the time is short. Apart from the discontinuities around the

(a)

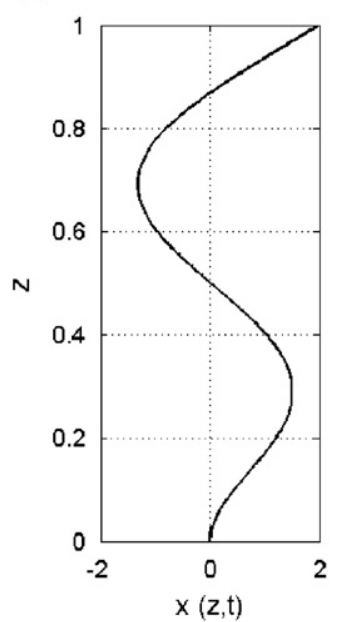

(b)

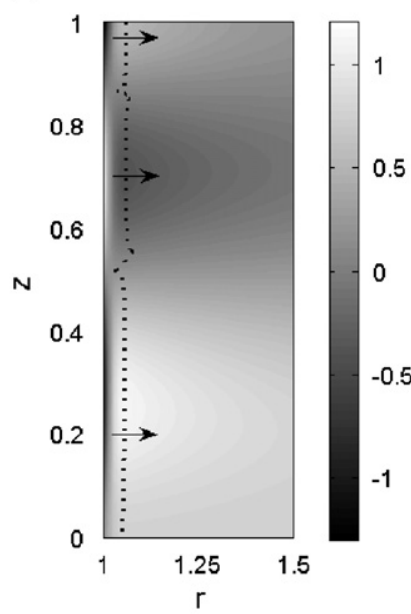

(c)

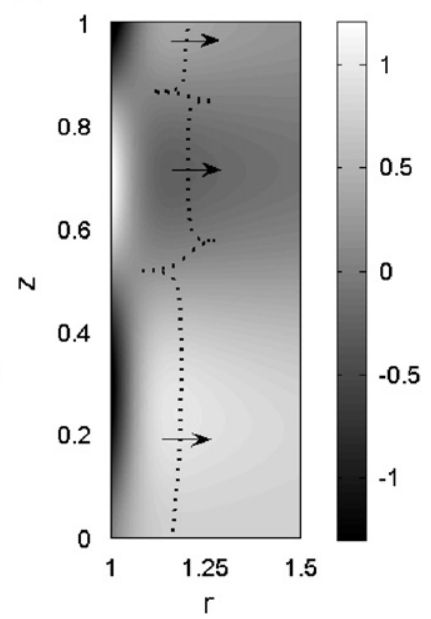

Fig. 3. (a) Third bending mode. (b) and (c) The kernel $Q_{3}(r, \theta, z, t)$ at $\theta=\pi / 2$, with $\alpha=2, \gamma=0.2$ and $\beta=500$. The dotted lines denote the position where the relative error between the viscous azimuthal velocity and that obtained with the potential flow model is one per cent. The arrows denote the waves propagation direction. 
points $z$ where the third eigenfunction equals zero (see Fig. 3(a)), the evanescent shear-wave propagation appears to be nearly the same for all $z$.

The integrated fluid forces can now be expressed. From symmetry considerations, only their $\mathbf{e}_{\mathbf{x}}$-components are nonzero. By keeping only the meaningful terms, i.e. those scaled after evaluation as $\mathcal{O}(1)$ and $\mathcal{O}\left(\beta^{1 / 2}\right)$, the dimensionless fluid forces are given by the formula

$$
F(z, t)=-\left.\frac{1}{\pi} \int_{0}^{2 \pi} p_{1}\right|_{r=1} \cos \theta+\left.\beta^{-1} \frac{\partial u_{\theta}}{\partial r}\right|_{r=1} \sin \theta \mathrm{d} \theta .
$$

The pressure term in the integral can be evaluated by performing an integration by part and substituting the azimuthal component of the momentum Eq. (22) in the resulting relation. The shear term in the integral can be obtained from Eq. (50). Once the dimensionless fluid forces are obtained, their projections on the $n$th flexure beam mode yield the modal fluid force coefficients:

$$
f_{n}(t)=\varphi_{n}^{0} \ddot{x}_{i}(t)-\sum_{m=1}^{\infty}\left[\sum_{q=0}^{\infty} \varphi_{m}^{q} \varphi_{n}^{q} c_{q}\right] \ddot{x}_{m}(t)-\frac{2}{\sqrt{\pi \beta}} \int_{0}^{t} \frac{1}{\sqrt{t-\tau}}\left\{\ddot{x}_{n}(\tau)+\sum_{m=1}^{\infty}\left[\sum_{q=0}^{\infty} \varphi_{m}^{q} \varphi_{n}^{q} c_{q}\right] \ddot{x}_{m}(\tau)\right\} \mathrm{d} \tau .
$$

The first two terms on right-hand side are the same as those obtained from the potential model, Eq. (46). They correspond to the contribution of the fluid part, which responds instantaneously to the boundary motions. The third term, inversely proportional to the square root of the Stokes number, is the first-order viscous rectification. It involves the total history of the relative beam motions through a convolution product. Its kernel, scaled as $t^{-1 / 2}$, indicates that a motion at time $\tau$ has decreasing influence on the fluid forces at time $t$ as $t-\tau$ increases. This reflects the decrease in shear and the loss of memory induced by propagation in the fluid domain of the evanescent viscous wave created at the beam surface as it is put into motion. The kernel $t^{-1 / 2}$, well known for a flat plate, can be found in classical textbooks (Basset, 1888; Lamb, 1932). The modal fluid force coefficients can also be put into the general form, Eq. (36):

$$
\begin{aligned}
& C_{i}^{n}(t)=\varphi_{n}^{0} \delta(t), \\
& C_{n}^{n}(t)=\delta(t) \sum_{q=0}^{\infty}\left(\varphi_{n}^{q}\right)^{2} c_{q}+\frac{2}{\sqrt{\pi \beta}} \frac{1}{\sqrt{t}}\left[1+\sum_{q=0}^{\infty}\left(\varphi_{n}^{q}\right)^{2} c_{q}\right], \\
& \left.C_{m}^{n}(t)=\delta(t)+\frac{2}{\sqrt{\pi \beta}} \frac{1}{\sqrt{t}}\right) \sum_{q=0}^{\infty} \varphi_{m}^{q} \varphi_{n}^{q} c_{q} \text { for } m \neq n .
\end{aligned}
$$

There is no viscous correction linked to the fluid response function Eq. (54) since this term corresponds to the irrotational motion imposed to the whole system, which does not create any flow. The contribution of the $m$ th mode to the $n$th mode involves both potential and viscous effects. Obviously, as $\beta \rightarrow \infty$, the viscous model solution tends to the potential one since a stable viscous boundary layer is assumed.

The beam response function $H_{1}(t)$ is illustrated in Fig. 2(b) for the dimensionless parameters $\mathscr{N}=1, \mathscr{M}=\frac{1}{8}, \alpha=2$, and $\gamma=0.2$ and different Stokes numbers $\beta$. The dotted line corresponds to the response function obtained with the potential model. In addition to the potential flow/elasticity interaction phenomena already described in Section 4.2, the response functions contain the influence of fluid viscosity. At early times, since the viscous shear wave is still very close to the boundary, the loss of energy induced by its propagation in the fluid domain is negligible and the curves coincide with those obtained with the potential model. As time increases, the evanescent waves are continuously generated on the boundary and dissipated as they propagate in the potential flow, which produces the damping effect. This phenomenon obviously occurs all the faster since the Stokes number is low.

\subsection{Interaction with an acoustic flow}

Assuming now that $\beta \rightarrow \infty, K=\mathcal{O}\left(\mathscr{M}_{a}\right)$ and $\Omega=\mathcal{O}(1)$, the flow is governed by the wave Eq. (35) for the velocity potential with the Neumann-type boundary conditions, Eq. (25). The vertical length and velocity are renormalized, respectively, by $L$ and $U_{i} R_{1} / L$ so as to be consistent with the beam normalization factors. The velocity potential is obtained by expressing the wave equation in a cylindrical coordinate system in the Laplace domain, using separation of 
variables and the relations (4) and (25):

$$
\left.\hat{\phi}_{0}(r, \theta, z, s)=\hat{c}_{i}(r, s) \hat{\dot{x}}_{i}(s) \cos \theta-\sum_{q=0}^{\infty} \hat{c}_{q}(r, s) \sum_{m=1}^{\infty} \varphi_{m}^{q} \hat{\dot{x}}_{m}(s)\right) \cos (q \pi z) \cos \theta,
$$

where the coefficients $\hat{c}_{i}(r, s)$ and $\hat{c}_{q}(r, s)$ take here the form

$$
\begin{aligned}
& \hat{c}_{i}(r, s)=\frac{1}{k_{0}} \frac{\left[\mathrm{Y}_{1}^{\prime}\left(k_{0} \alpha\right)-\mathrm{Y}_{1}^{\prime}\left(k_{0}\right)\right] \mathrm{J}_{1}\left(k_{0} r\right)-\left[\mathrm{J}_{1}^{\prime}\left(k_{0} \alpha\right)-\mathrm{J}_{1}^{\prime}\left(k_{0}\right)\right] \mathrm{Y}_{1}\left(k_{0} r\right)}{\mathrm{J}_{1}^{\prime}\left(k_{0}\right) \mathrm{Y}_{1}^{\prime}\left(k_{0} \alpha\right)-\mathrm{J}_{1}^{\prime}\left(k_{0} \alpha\right) \mathrm{Y}_{1}^{\prime}\left(k_{0}\right)}, \\
& \hat{c}_{q}(r, s)=-\frac{\Delta_{q}}{k_{q}} \frac{\mathrm{Y}_{1}^{\prime}\left(k_{q} \alpha\right) \mathrm{J}_{1}\left(k_{q} r\right)-\mathrm{J}_{1}^{\prime}\left(k_{q} \alpha\right) \mathrm{Y}_{1}\left(k_{q} r\right)}{\mathrm{J}_{1}^{\prime}\left(k_{q}\right) \mathrm{Y}_{1}^{\prime}\left(k_{q} \alpha\right)-\mathrm{J}_{1}^{\prime}\left(k_{q} \alpha\right) \mathrm{Y}_{1}^{\prime}\left(k_{q}\right)},
\end{aligned}
$$

with $\Delta_{q}=1$ for $q=0$ and $\Delta_{q}=2$ for $q \geqslant 1$. The prime denotes the derivative according to the function argument, $\mathrm{J}_{1}$ and $\mathrm{Y}_{1}$ are the Bessel function of order 1 of the first and second kind, respectively (Abramowitz and Stegun, 1970), and $k_{q}(s)$ is defined by $k_{q}(s)=\mathrm{i}\left[(\Omega s)^{2}+(q \pi \gamma)^{2}\right]^{1 / 2}$. The first-order fluid pressure can then be deduced from the Bernoulli relation, Eq. (26), and evaluated in the temporal domain by using the convolution theorem:

$$
p_{1}(r, \theta, z, t)=\int_{0}^{t} K_{i}(r, \theta, t-\tau) \ddot{x}_{i}(\tau) \mathrm{d} \tau+\sum_{m=1}^{\infty} \int_{0}^{t} K_{m}(r, \theta, z, t-\tau) \ddot{x}_{m}(\tau) \mathrm{d} \tau
$$

where the kernels $K_{i}(r, \theta, t)$ and $K_{m}(r, \theta, z, t)$ are the fluid pressure responses induced by the impulsional motion, respectively, of the whole system and of the $m$ th flexure beam mode:

$$
K_{i}(r, \theta, t)=-c_{i}(r, t) \cos \theta, \quad K_{m}(r, \theta, z, t)=\sum_{q=0}^{\infty} \varphi_{m}^{q} c_{q}(r, t) \cos (q \pi z) \cos \theta,
$$

with $c_{i}(r, t)$ and $c_{q}(r, t)$ the inverse Laplace transforms of $\hat{c}_{i}(r, s)$ and $\hat{c}_{q}(r, s)$. Their analytical expressions are not straightforward, but they can be efficiently evaluated by a numerical inverse of the Laplace transform. The kernel $K_{2}(r, \theta, z, t)$ is illustrated in Fig. 4 at $\theta=0$ with dimensionless numbers $\alpha=2, \Omega=1$ and $\gamma=0.2$. It appears from Eq. (60) that all the fluid vertical modes are involved. At $t=0$, the impulsional motion of the second bending mode, Fig. 4(a), generates a pressure wave on the inner cylinder boundary that consists of both a compression and a rarefaction wave, depending on the sign of $\varphi_{2}(z)$ along the vertical coordinate $z$. This case is equivalent to a problem in which both the radial clearance and the propagation velocities are equal to one. So the pressure wave is reflected back on the boundaries at each $t=k, k \in \mathbb{N}$. This phenomenon recurs indefinitely since the acoustic model in a confined fluid domain does not contain any damping mechanism.

(a)

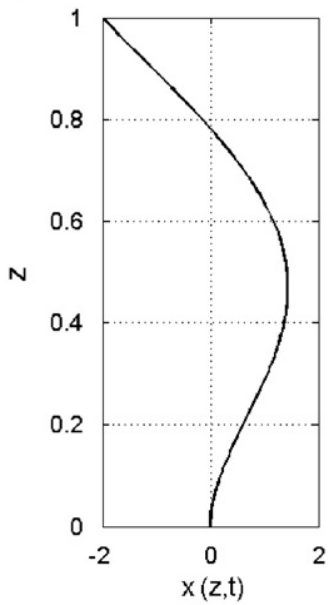

(b)

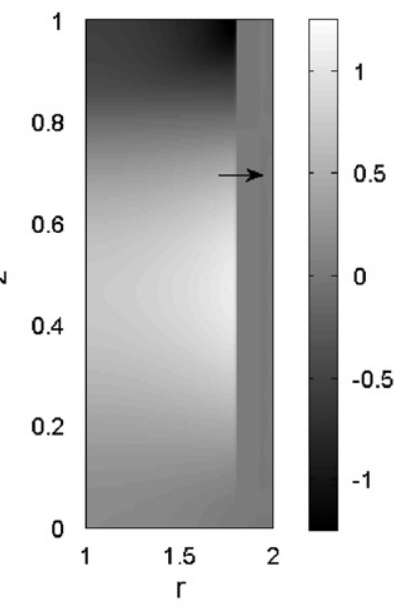

(c)

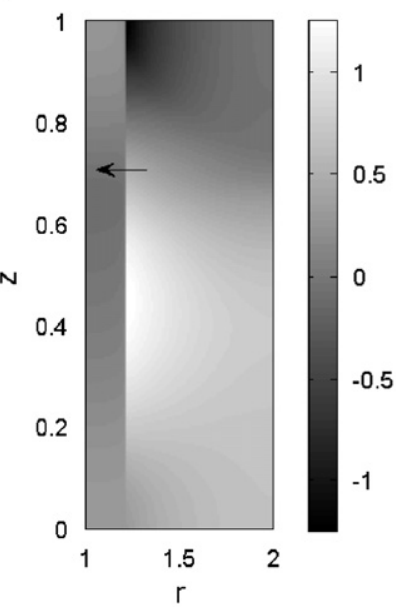

Fig. 4. (a) Second bending mode. (b) and (c) The kernel $K_{2}(r, \theta, z, t)$ at $\theta=0$, with $\alpha=2, \gamma=0.2$ and $\Omega=1$. The arrows denote the waves propagation direction. 
(a)

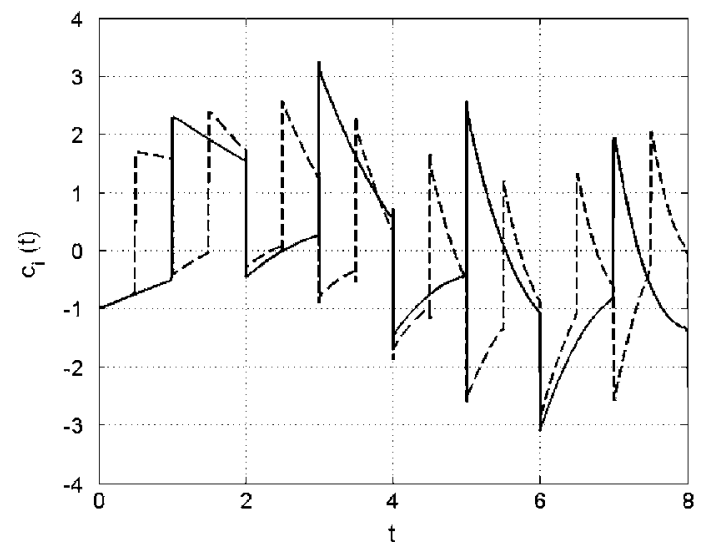

(b)

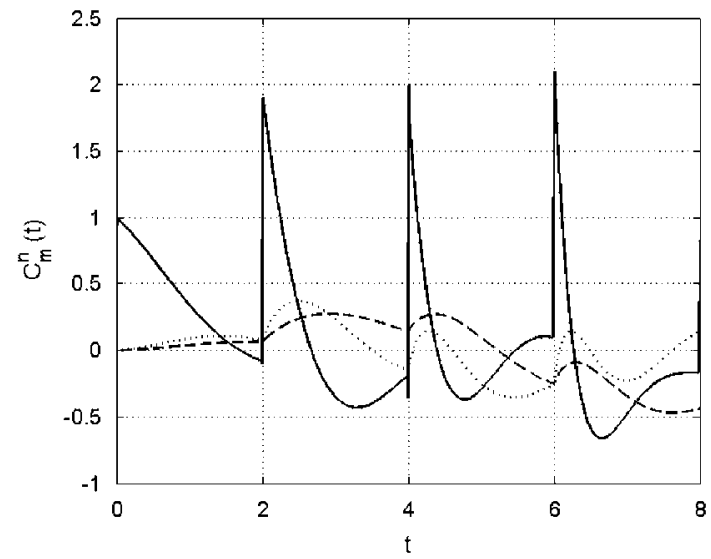

Fig. 5. Time-dependent coefficients of the modal fluid forces. (a) Influence of the impulsional motion of the whole system for $\Omega=1$ and different $\alpha$ :,$- \alpha=2 ;--, \alpha=1.5$. (b) Influence of the impulsional motion of the first, second and third bending modes on the second mode, for $\Omega=1, \alpha=2$ and $\gamma=0.2$. -,$C_{2}^{2}(t) ;--, C_{1}^{2}(t)$ and $\cdots, C_{3}^{2}(t)$.

The expression for the fluid forces on the beam surface at a given $z$ can be deduced by integrating the fluid pressure Eq. (59) according to $\theta$. Only its $\mathbf{e}_{\mathbf{x}}$-direction component is nonzero and its projection on the $n$th flexure beam mode yields the expression for the modal fluid force coefficients,

$$
f_{n}(t)=\varphi_{n}^{0} \int_{0}^{t} c_{i}(t-\tau) \ddot{x}_{i}(\tau) \mathrm{d} \tau-\sum_{m=1}^{\infty} \int_{0}^{t}\left[\sum_{q=0}^{\infty} \varphi_{m}^{q} \varphi_{n}^{q} c_{q}(t-\tau)\right] \ddot{x}_{m}(\tau) \mathrm{d} \tau,
$$

where $c_{i}(t)=c_{i}(1, t)$ and $c_{q}(t)=c_{q}(1, t)$. These forces can also be expressed into the general form, given by Eq. (36), as

$$
C_{i}^{n}(t)=\varphi_{n}^{0} c_{i}(t) \quad \text { and } \quad C_{m}^{n}(t)=\sum_{q=0}^{\infty} \varphi_{m}^{q} \varphi_{n}^{q} c_{q}(t)
$$

The coefficients for the modal fluid forces associated with the impulsional motion of the whole system, $C_{i}^{n}(t)$, differ from each other by the geometrical constant $\varphi_{n}^{0}$, and they can therefore be described through the analysis of $c_{i}(t)$ only. This function is displayed in Fig. 5(a) for $\Omega=1$ and different $\alpha$. These curves have points of finite discontinuity that account for the reflections of pressure waves in the fluid domain. For instance, for $\Omega=1$ and $\alpha=2$, the compression wave induced by the impulse motion of the inner cylinder at $t=0$ is reflected back on the outer cylinder at $t=1$ and influences the integrated fluid forces on the inner cylinder at $t=2$. During this time, the rarefaction wave generated by the impulse motion of the outer cylinder at $t=0$ arrives on the inner cylinder at $t=1$ and creates a discontinuity in the fluid forces. Hence, the time interval between the discontinuities is equal to half the time for a wave to travel back and forth from one cylinder to the other. Furthermore, the more confined the fluid domain, the more discontinuities the kernel contains. At the beginning of the process, that is before the rarefaction wave comes into contact with the beam surface, the kernel is seen to be independent of the confinement ratio, a consequence of the finite propagation velocity: the inner cylinder is not "informed" yet of the presence of the outer boundary. In addition to the finite discontinuities, the curves in Fig. 5(a) display a low frequency modulation, nearly independent of the confinement ratio. Deriving the power spectrum of $c_{i}(t)$ for $\Omega=1$ shows that the frequency of this modulation tends to 1 as $\alpha \rightarrow 1$, which is exactly the frequency linked to the time for a wave of velocity 1 to cover a distance of $2 \pi$. Therefore, the low-frequency modulation is associated to azimuthal propagation of pressure waves.

The time-dependent modal coefficients $C_{m}^{n}(t)$, illustrated in Fig. 5(b) for $n=2$ and $m=1,2$ and 3, represent the contribution of the impulsional motion of the first, second and third bending modes on the fluid forces felt by the second one. The curve of $C_{2}^{2}(t)$ displays finite discontinuities due to the multiple reflections of the pressure wave generated at $t=0$ by the impulsional motion of the second bending mode. The interval between the discontinuities is equal to the time for this wave to travel back and forth from the beam to the outer cylinder. The other curves, namely $C_{1}^{2}(t)$ and $C_{3}^{2}(t)$, are more regular and only their time derivatives contain discontinuities. They are equal to zero at $t=0$ and their contributions remain negligible at early times. This behaviour is a consequence of the in vacuo eigenfunctions orthogonality: the wave generated at $t=0$ by the impulsional motion of the $n$th bending mode has the shape of this 
(a)

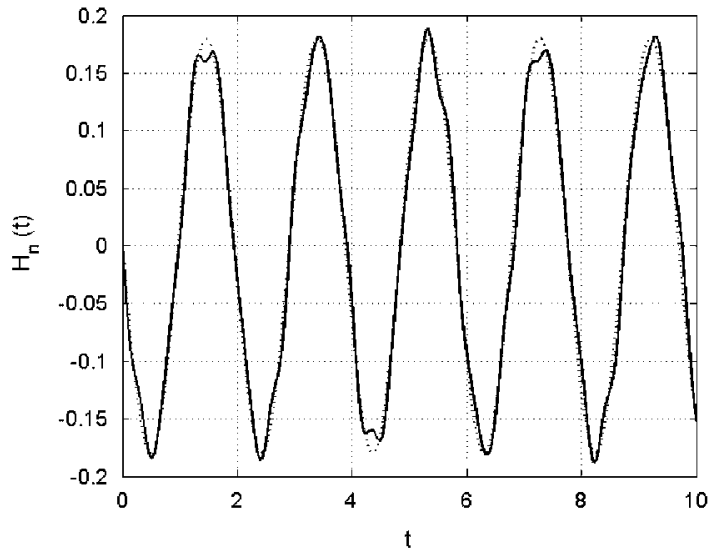

(b)

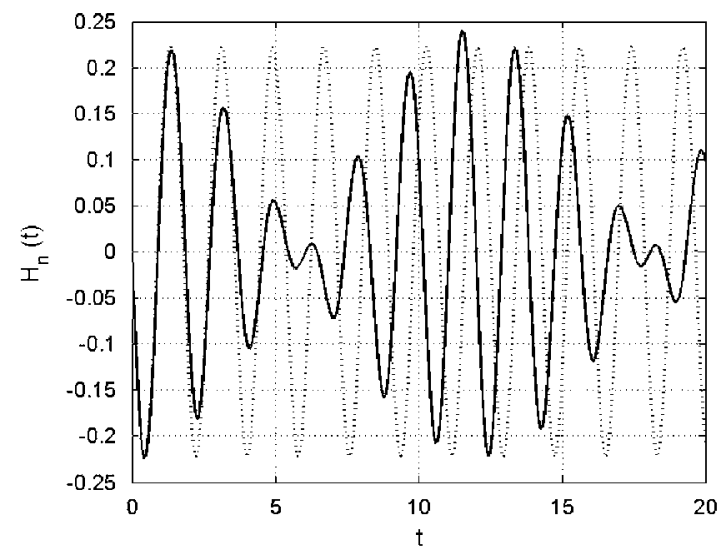

Fig. 6. The first impulse function $H_{1}(t)$ obtained with the acoustic model-for the dimensionless parameters $\mathscr{W}=1, \mathscr{M}=\frac{1}{8}, \alpha=2$, $\gamma=0.2$ and different compressibility numbers $\Omega$ : (a),$- \Omega=0.05 ; \cdots$, potential model and (b),$- \Omega=1 ; \cdots$, in vacuo model.

mode. Hence its influence on the other modes is null, they are uncoupled. As the wave propagates in the cylindrical domain, it loses its initial shape with the result that its projection on the other modes becomes significant, they are fully coupled. Unlike the viscous case, in order to evaluate the fluid forces at a time $t$, the motion of the boundaries at a previous time $\tau$ is as important as that at time $t$, even though $t-\tau$ is large: there is no loss of fluid memory, since the outgoing pressure waves are reflected back and are not affected by any damping mechanism.

The beam response function $H_{1}(t)$ is illustrated in Fig. 6 for the dimensionless parameters $\mathscr{W}=1, \mathscr{M}=\frac{1}{8}, \alpha=2$, $\gamma=0.2$ and different compressibility numbers $\Omega$. For $\Omega=0.05$, Fig. 6(a), the frequencies linked to the pressure-wave propagation in the fluid domain are above that of the first bending mode, and hence the response function is close to that obtained with the potential model, with only a high-frequency modulation due to the multiple rebounds of the pressure waves. On the other hand, for $\Omega=1$ (see Fig. 6(b)), the first frequency linked to the pressure wave propagation is lower than that of the first bending mode. Hence at early times, the fluid has not had the time to manifest itself and the bending mode, not informed of the fluid presence, responds as if in vacuo. As time increases, an elasticity-compressibility coupling appears, resulting in a low-frequency modulation of the curve.

\section{The models for an imposed sine-wave shock}

\subsection{Sine-wave shock}

The fluid-structure interaction models obtained in Section 4 are now illustrated and compared for a specific motion imposed on the whole system. This motion consists of an unique sinusoidal period of acceleration. Their dimensional and dimensionless expressions (using the normalization factors in Table 1(a)) take, respectively, the form,

$$
\tilde{\ddot{x}}_{i}(\tilde{t})=\left\{\begin{array}{ll}
\frac{U_{i} \omega_{i}}{2} \sin \omega_{i} \tilde{t} & \forall \tilde{t} \in\left[0,2 \pi / \omega_{i}\right], \\
0 & \forall \tilde{t}>2 \pi / \omega_{i},
\end{array} \quad \ddot{x}_{i}(t)= \begin{cases}\frac{1}{2} \sin t & \forall t \in[0,2 \pi], \\
0 & \forall t>2 \pi,\end{cases}\right.
$$

where $U_{i}$ and $\omega_{i}$ are the characteristic velocity and frequency of the shock. Hence the maximum acceleration is given by $U_{i} \omega_{i} / 2$ and its dimensionless expression is $\frac{1}{2}$. Even though this function is partly composed of a harmonic function, it can be considered a purely transient motion since it starts from rest at $t=0$ and stops at $t=2 \pi$. It consists of a simple imposed translation and is representative of the early residual motion undergone by equipment during shock loading (Keil, 1961; O'Hara and Cunnif, 1993). Employing usual Laplace transform relations, its expression in the Laplace domain is given by

$$
\hat{\ddot{x}}_{i}(s)=\frac{1-\mathrm{e}^{-2 \pi s}}{2\left(s^{2}+1\right)} .
$$


Substituting this relation in the truncated system, Eq. (39), performing the numerical matrix and Laplace transform inversions, Eq. (40), and doing the modal recomposition, Eq. (41), yield the dimensionless relative displacement $x(z, t)$. From the design point of view, knowledge of the absolute beam acceleration, $\ddot{x}_{a}(z, t)$, is also useful. Its dimensionless form is derived from the relative beam displacement thanks to the relation $\ddot{x}_{a}(z, t)=\ddot{x}(z, t)+\ddot{x}_{i}(t)$. Since the parameter space $(\mathscr{M}, \mathscr{W}, \alpha, \gamma, \beta, \Omega)$ is quite large, all the system characteristics cannot be easily assessed and synthesized, so only a few general behaviours are illustrated below.

\subsection{Variation of the beam as a function of the dimensionless frequency $\mathscr{W}$}

From the design point of view, the maximum absolute acceleration experienced by the shock-loaded structures as a function of the system stiffness is of interest. For a clamped-free beam, the maximum occurs at its free end, i.e. $z=1$. Hence, the variation of the maximum absolute beam acceleration, $\max _{t}\left|\ddot{x}_{a}(1, t)\right|$, normalized by the maximum imposed acceleration, i.e. $\frac{1}{2}$, as a function of $\mathscr{W}$ is particularly meaningful. Such curves are shown in Fig. 7(a) for a beam in vacuo and in a potential flow with dimensional parameters $\alpha=2, \gamma=0.2$ and $\mathscr{M}=\frac{1}{8}$. For large $\mathscr{W}$, the beam is stiff in relation to the imposed shock, so the relative displacement tends to zero and the maximum absolute acceleration tends to that imposed on the whole system. As $\mathscr{W}$ decreases, pseudo-resonances between the imposed shock frequency and the characteristic elastic beam frequencies are observed. The mean influence of the potential flow is to reduce significantly the amplitude of the peaks as a result of inertial and added-mass effects. While the maximum acceleration in vacuo, encountered for the first bending mode pseudo-resonance, is more than five times that of the imposed shock, it is reduced to barely four times with a potential flow for $\mathscr{M}=\frac{1}{8}$. Unlike the case of a single-degree-of-freedom spring-mass system in vacuo, where the maximum acceleration tends rapidly to zero as the spring stiffness tends to zero (Lalanne, 1999), here the pseudo-resonance with each bending mode prevent this rapid decrease as $\mathscr{W} \rightarrow 0$.

The maximum absolute beam accelerations obtained with the viscous model is illustrated in Fig. 7(b) for $\alpha=2$, $\gamma=0.2, \mathscr{M}=\frac{1}{8}$ and $\beta=1000$. The main influence of viscosity is to slightly reduce the amplitude of the pseudoresonance peaks in relation to those obtained with the potential model. This decrease is not always the same: for instance, the fifth bending mode pseudo-resonance is almost unaffected whereas the fourth is greatly reduced in relation to the first three. The reason for these discrepancies lies in the different times at which the peaks are attained: the influence of viscosity is more pronounced as the maximum is reached at large time.

The influence of fluid compressibility is illustrated for $\Omega=1$ in Fig. 8(a) and for $\Omega=0.4$ in Fig. 8(b), with $\alpha=2$, $\gamma=0.2$ and $\mathscr{M}=\frac{1}{8}$. As in the viscous case, no meaningful difference from the potential model can be observed for large $\mathscr{W}$. On the contrary, the curves display contrasting behaviours in the resonant zone. For $\Omega=1$, all peaks are higher than those obtained in vacuo. Furthermore, the high-frequency in vacuo flexure modes cannot be clearly identified in a compressible flow, since they are fully coupled with the acoustic waves: the pseudo-resonant peaks occur near the characteristic frequencies of the coupled elastic acoustic system. For $\Omega=0.4$, the maximum acceleration is lower than that obtained with a potential flow, while the other peaks are slightly higher. Further numerical simulations

(a)

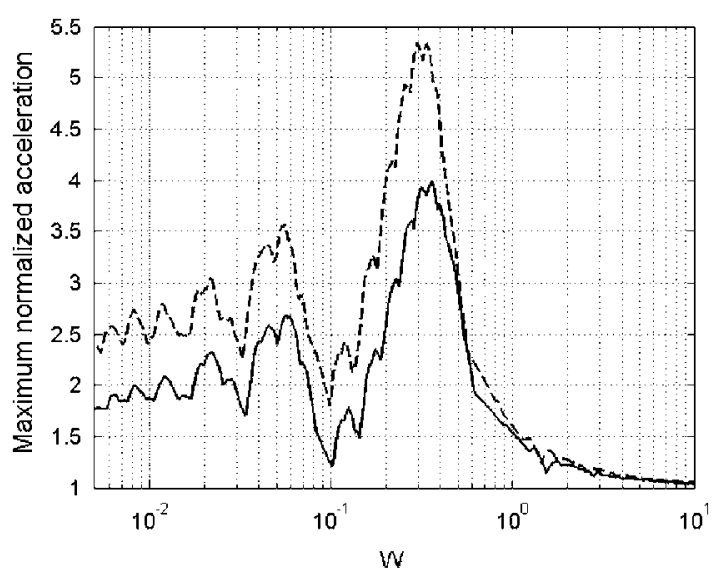

(b)

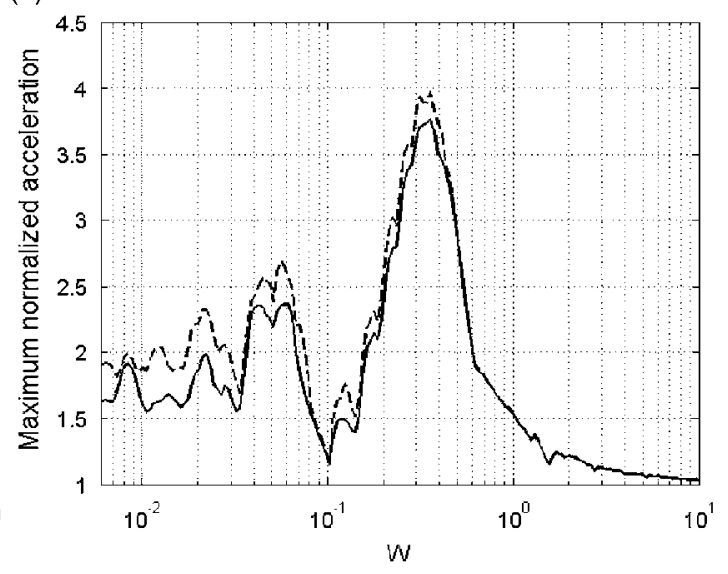

Fig. 7. Maximum absolute beam acceleration $\left|\ddot{x}_{a}(z, t)\right|$ at $z=1$, normalized by the maximum imposed acceleration, for $\alpha=2, \gamma=0.2$, $\mathscr{M}=\frac{1}{8}$, as a function of the dimensionless frequency $\mathscr{W}:$ (a) - - , in vacuo model; - , potential model and (b) - - , potential model, $\beta=1000$. 
(a)

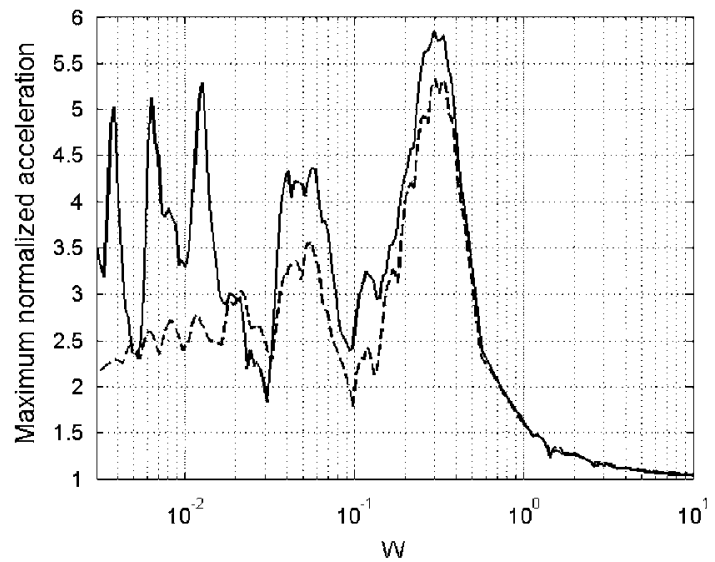

(b)

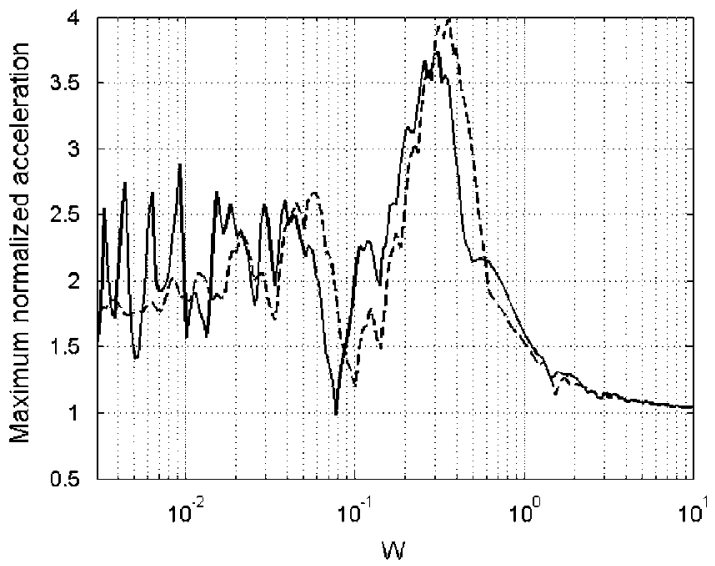

Fig. 8. Maximum absolute beam acceleration $\left|\ddot{x}_{a}(z, t)\right|$ at $z=1$, obtained with the in vacuo, potential and acoustic models, normalized by the maximum imposed acceleration, for $\alpha=2, \gamma=0.2$ and $\mathscr{M}=\frac{1}{8}$, as a function of the dimensionless frequency $\mathscr{W}:$ (a) -, $\Omega=1 ;---$, in vacuo model and (b) -, $\Omega=0.4 ;---$, potential model.

(a)

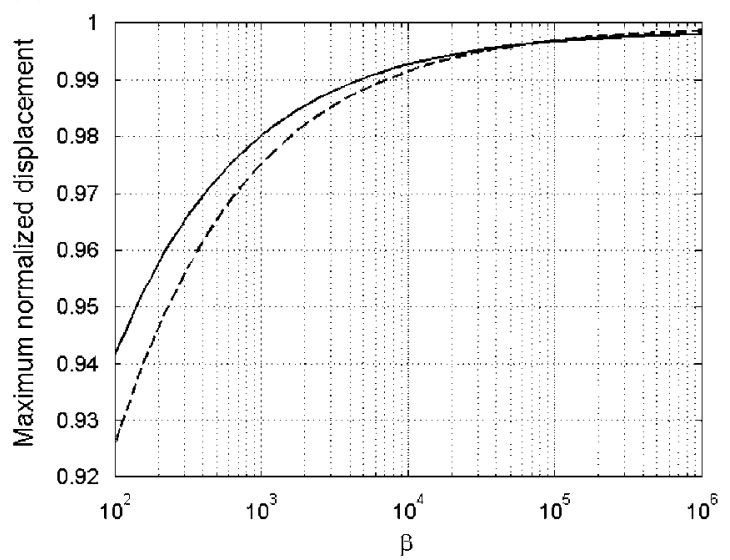

(b)

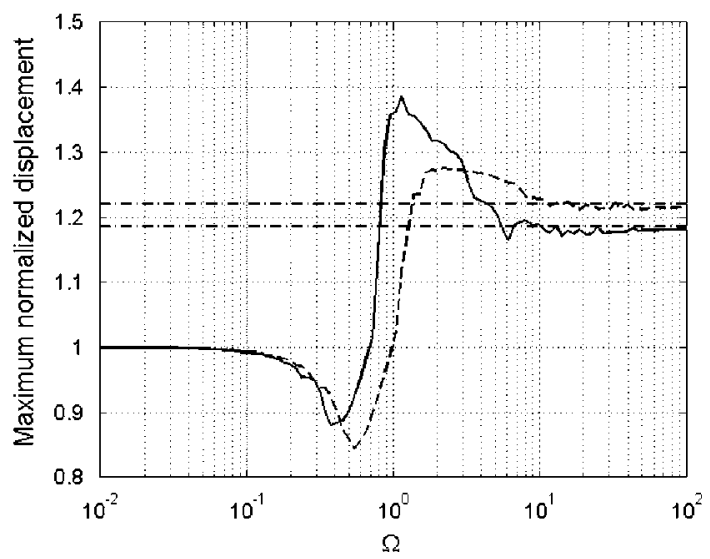

Fig. 9. Maximum nondimensional relative beam displacement $|x(z, t)|$ at $z=1$ normalized by the maximum value of the potential model, for $\gamma=0.2, \mathscr{M}=\frac{1}{8}, \mathscr{W}=0.3$ and different confinement numbers:,$- \alpha=2 ;--, 1.5$ : (a) with the viscous boundary-layer model as a function of the Stokes number and (b) with the acoustic model as a function of the compressibility number. The dash-dot lines -.denote the maximum normalized displacement in vacuo.

for different $\Omega$ show other characteristics, with the result that it may be difficult to extract general trends, and they must be analysed individually. Some elements can nevertheless be obtained by looking at the variation of the maximum relative displacement as a function of the compressibility number at a fixed $\mathscr{W}$.

\subsection{Variation of the beam response as a function of the stokes and compressible numbers}

The response of the fluid-structure system to a sine-wave shock can also be studied by analysing the maximum relative displacement, $\max _{t}|x(z, t)|$ at $z=1$, as a function of the Stokes and compressibility numbers for fixed $\alpha, \gamma, \mathscr{M}$ and $\mathscr{W}$, and normalized by the maximum value obtained with a potential flow. This yields a complementary view of the system behaviour and highlights the influence of fluid viscosity and compressibility. Such curves are given in Fig. 9 for $\gamma=0.2, \mathscr{M}=\frac{1}{8}, \mathscr{W}=0.3$, and $\alpha=2$ and 1.5 . Note that $\mathscr{W}=0.3$ corresponds approximately to the highest pseudoresonant peak in Figs. 7 and 8. For the viscous case, Fig. 9(a), the maximum displacement is smaller than that obtained 


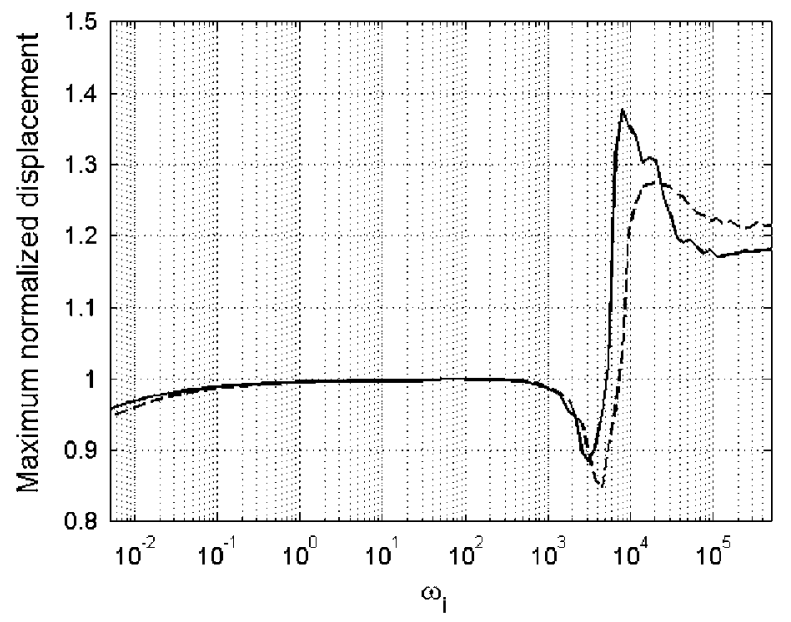

Fig. 10. Maximum relative beam displacement $|x(z, t)|$ at $z=1$, obtained with the viscous and acoustic models and normalized by the maximum of the potential model, as a function of the dimensional imposed motion frequency $\omega_{i}$. The parameters are $R_{1}=0.2 \mathrm{~m}$, $v=10^{-6} \mathrm{~m}^{2} \mathrm{~s}^{-1}, c_{f}=1500 \mathrm{~m}^{2} \mathrm{~s}^{-1}, \gamma=0.2, \mathscr{M}=\frac{1}{8}, \mathscr{W}=0.3$ and,$- \alpha=2 ; \cdot \cdot-\cdot, \alpha=1.5$.

with a potential flow. Obviously, this effect becomes more pronounced as the Stokes number decreases. As $\beta \geqslant 4 \times 10^{4}$, the viscous rectification is less than $0.5 \%$ of that for the potential flow. The acoustic case is illustrated in Fig. 9 (b). For low compressibility numbers, $\Omega<0.1$, the maximum displacements tend to the maximum potential values while for high compressibility numbers, $\Omega>10$, they tend to the values obtained without fluid. Between these two limits, as $\Omega$ increases, the maximum relative displacements decrease regularly to a minimum well below the potential value and lower for $\alpha=1.5$ than for $\alpha=2$. Then, the maximum relative displacements show a sharp increase to a peak well above the maximum value obtained without fluid and more pronounced for $\alpha=2$.

\subsection{Use of the models}

The viscous and compressible models, described independently in the previous section as functions of their corresponding dimensionless numbers, can be grouped on a single figure for a particular geometry. Hence the significant fluid effects in a particular case can be identified. For instance, by fixing the dimensional parameters $R_{1}, v$ and $c_{f}$, and the dimensionless numbers $\gamma, \alpha, \mathscr{M}$ and $\mathscr{W}$, the maximum relative displacements, $\max _{t}|x(z, t)|$ at $z=1$, can be displayed as a function of the dimensional shock frequency $\omega_{i}$. Since $\mathscr{W}$ is defined by $\omega_{i}^{-1}\left(E I / \rho_{s} S L^{4}\right)^{1 / 2}$, the quantity $\left(E I / \rho_{s} S L^{4}\right)^{1 / 2}$ must be adjusted for each $\omega_{i}$ so as to maintain $\mathscr{W}$ constant. Hence, increasing $\omega_{i}$ makes the Stokes number and the compressibility number to increase. Such curves are given in Fig. 10 with $R_{1}=0.2 \mathrm{~m}, v=10^{-6} \mathrm{~m}^{2} \mathrm{~s}^{-1}$, $c_{f}=1500 \mathrm{~m} \mathrm{~s}^{-1}, \gamma=0.2, \alpha=1.5$ and $2, \mathscr{M}=\frac{1}{8}$ and $\mathscr{W}=0.3$. For $\omega_{i}<50 \mathrm{~s}^{-1}$, the curves are obtained with the viscous fluid model, and with the acoustic one for $50 \mathrm{~s}^{-1}<\omega_{i}$. All the curves are then normalized with the maximum displacement obtained with the potential fluid model (which is independent of the frequency $\omega_{i}$ ) for each confinement ratio. A smooth transition is observed between the curves obtained with the viscous and acoustic fluid models. It constitutes a check of the correctness of the derivation, in which each effect are treated independently. From this single graph, the bounds of the ranges corresponding to the three regimes can be defined subjectively. When the curve tends to unity, this means that the flow is dominated by potential effects. The bounds are defined such that deviations from unity exceeds approximately $2 \%$. This occurs for the viscous model when $\omega_{i} \leqslant 10^{-1} \mathrm{~s}^{-1}$ and for the acoustic one when $10^{3} \mathrm{~s}^{-1} \leqslant \omega_{i}$. Therefore, for imposed frequencies such that $\omega_{i} \leqslant 10^{-1} \mathrm{~s}^{-1}$, viscous effects must be taken into account, for $10^{-1} \mathrm{~s}^{-1} \leqslant \omega_{i} \leqslant 10^{3} \mathrm{~s}^{-1}$ the flow is dominated by potential effects and for $10^{3} \mathrm{~s}^{-1} \leqslant \omega_{i}$, fluid compressibility must be considered. Thus, a meaningful fluid model can be easily identified on a broad range of motions.

\section{Conclusions}

This paper has presented a semi-analytical approach to the study of an elastic circular cylinder confined in a cylindrical fluid domain initially at rest and subjected to small-amplitude transient motions. The elastic flexure beam 
model is chosen for the circular cylinder dynamics and the transverse beam displacement is expanded on the in vacuo sets of eigenvectors, with time-dependent coefficients. The three fluid models, potential, viscous boundary-layer and acoustic, are derived for small Mach numbers $\mathscr{M}_{a}$ by means of perturbation methods so as to highlight their links and limits a priori. Hence, the potential flow model is expected to be valid in the range of parameters $\beta \rightarrow \infty, \mathscr{M}_{a}^{2} \ll K \ll 1$ and $\Omega \ll 1$, the viscous boundary layer model for $\beta^{\frac{1}{2}}(\alpha-1) \gg 1, \mathscr{M}_{a}^{2} \ll K \ll 1$ and $\Omega \ll 1$, and the acoustic model for $\beta \rightarrow \infty, K=\mathcal{O}\left(\mathscr{M}_{a}\right)$ and $\Omega=\mathcal{O}(1)$. The resolution method for the fluid-structure interaction problem is then presented, with the same approach for the three fluid models. The problem is turned into an infinite number of fully coupled integro-differential equations involving the modal time-dependent displacement coefficients. It is solved, after truncation, by matrix inversions in the Laplace domain. A fast numerical inversion of the Laplace transform can then be employed to return to the temporal domain. The governing equations of the fluid medium are then solved for each fluid model by separation of variables. The solutions, put into the form of convolution products, are expressed for arbitrary motions of the domain boundaries. Analysis of their kernels yields a physical understanding of the fluid behaviour and highlights the influence of the related wave-propagation phenomena on the fluid forces. The response functions, involving both the wave-propagation phenomena in the fluid domain and the beam elasticity, are also introduced and described. Then, the fluid-structure interaction solutions are illustrated for a specific imposed motion, a sine-wave shock, that is representative of the early residual motion felt by equipments on board of surface ships or submarines subjected to the effects of underwater explosion. Some general behaviour is illustrated by displaying the maximum absolute beam acceleration as a function of the dimensionless frequency $\mathscr{W}$ and the maximum relative beam displacement as a function of the Stokes and compressibility numbers. Last, the models are examined simultaneously for a particular case and are shown to cover a broad range of motions. Thus for a given geometry and displacement imposed on the whole system, a meaningful fluid model can be identified.

These models emphasize some fluid-structure interaction effects in simple asymptotic cases. However, not all physical phenomena are, of course, treated here. For instance, large-relative-displacement beam motions are not considered, so that boundary-layer separation issues are not handled. Furthermore, the viscous flow is assumed stable, and thus centrifugal and transient hydrodynamic instabilities are not taken into account. Another restriction comes from the single-phase assumption, which makes the models incapable of taking cavitation phenomena into account. The present article yields, however, converged three-dimensional fluid-structure solutions that can be used for estimating the shockloaded structures displacement in the first step of design studies and, if required, for selecting a meaningful fluid model for further investigations. They are also believed to be suitable for validating more evolved numerical codes that can solve fluid-structure interaction problems in more complex geometries.

\section{References}

Abramowitz, M., Stegun, I.A., 1970. Handbook of Mathematical Functions. Dover, New York.

Axisa, F., 2001. Modélisation des Systèmes Mécaniques. Milieux Continus. Hermès, Paris.

Basset, A.B., 1888. A Treatise on Hydrodynamics, vol. 1. Deighton, Bell and Co., Cambridge.

Blevins, R.D., 1984. Formulas for Frequency and Mode Shape. Robert E. Kreiger Publishing Company, New York.

Brancik, L., 2001. Utilization of quotient-difference algorithm in FFT based numerical ILT method. In: Proceedings 11th Radioelektronica 2001, Brno, Czech Republic, pp. 352-355.

Brancik, L., 2003. Utilization of Matlab in simulation of linear hybrid circuits. Radioengineering 12, 6-11.

Chen, S.S., 1987. Flow Induced Vibrations. Hemisphere Publishing Corporation, Washington, DC.

Van Dyke, M., 1964. Perturbation Methods in Fluid Mechanics. Academic Press, New York.

Gibert, R.J., 1986. Vibrations des Structures, Interaction avec les Fluides, Sources d'Excitations Aléatoires. Collection de la Direction des Etudes et Recherches d'Electricité de France, vol. 69. Eyrolles, Paris.

Habault, D., Filippi, P.J.T., 2003. On the transient response of a fluid-loaded structure represented by a series of resonances modes. Journal of Sound and Vibration 259, 1269-1275.

Habault, D., Filippi, P.J.T., 2004. A numerical method for the computation of the resonance frequencies and modes of a fluid-loaded plate: application to the transient response of the system. Journal of Sound and Vibration 270, 207-231.

Hall, P., 1984. On the stability of an unsteady boundary layer on a cylinder oscillating transversely in a viscous flow. Journal of Fluid Mechanics 146, 337-367.

Honji, H., 1981. Streaked flow around an oscillating circular cylinder. Journal of Fluid Mechanics 107, 507-520.

Iakovlev, S., 2002. Interaction of a spherical shock wave and a submerged fluid-filled circular cylindrical shell. Journal of Sound and Vibration 255, 615-633.

Iakovlev, S., 2004. Influence of a rigid coaxial core on the stress-strain state of a submerged fluid-filled circular cylindrical shell subjected to a shock wave. Journal of Fluids and Structures 19, 957-984.

Iakovlev, S., 2006. External shock loading on a submerged fluid-filled cylindrical shell. Journal of Fluids and Structures 22, 997-1028 
Iakovlev, S., 2007. Submerged fluid-filled cylindrical shell subjected to a shock wave: fluid-structure interaction effects. Journal of Fluids and Structures 23, 117-142.

Keil, A.H., 1961. The response of ships to underwater explosions. Transactions, Society of Naval Architects and Marine Engineers 69, 366-410.

Kevorkian, J., Cole, J.D., 1996. Multiple Scale and Singular Perturbation Methods. Springer, New York.

Keulegan, G.H., Carpenter, L.H., 1958. Forces on cylinders and plates in an oscillating fluid. Journal of Research of the National Bureau of Standards 60, 423-440.

Koumoutsakos, P., Leonard, A., 1986. High resolution simulations of the flow around an impulsively started circular cylinder using vortex methods. Journal of Fluid Mechanics 296, 1-38.

Lalanne, C., 1999. Vibrations et Chocs Mécaniques, Tome 2. Hermès, Paris.

Lamb, H., 1932. Hydrodynamics, sixth ed. Cambridge University Press, Cambridge.

Landau, L.A., Lifshitz, E.M., 1959. Fluid Mechanics. Pergamon Press, Oxford.

O'Hara, G.J., Cunnif, P.F., 1993. Scaling for shock response of equipment in different submarines. The Shock and Vibration Bulletin $1,161-170$.

Riley, N., 2001. Steady streaming. Annual Reviews of Fluid Mechanics 33, 43-65.

Sarpkaya, T., 1986. Force on a circular cylinder in viscous oscillatory flow at low Keulegan-Carpenter numbers. Journal of Fluid Mechanics 165, 61-71.

Sarpkaya, T., 2001. Hydrodynamic damping and quasi-coherent structures at large Stokes numbers. Journal of Fluids and Structures $15,909-928$.

Sarpkaya, T., 2002. Experiments on the stability of sinusoidal flow over a circular cylinder. Journal of Fluid Mechanics 457, 157-180.

Schlichting, H., 1979. Boundary-Layer Theory, seventh ed. McGraw-Hill, New York.

Schmid, P.J., Henningson, D.S., 2001. Stability and Transition in Shear Flows. Springer, New York.

Sigrist, J.-F., Broc, D., 2007. Homogenisation method for the modal analysis of a nuclear reactor with internal structures modelling and fluid-structure-interaction coupling. Nuclear Engineering and Design 237, 431-440.

Sigrist, J.-F., Broc, D., Lainé, C., 2006a. Dynamic analysis of a nuclear reactor with fluid-structure interaction. Part I: seismic loading, fluid added mass and added stiffness effects. Nuclear Engineering and Design 236, 2431-2443.

Sigrist, J.-F., Broc, D., Lainé, C., 2006b. Dynamic analysis of a nuclear reactor with fluid-structure interaction. Part II: shock loading, influence of fluid compressibility. Nuclear Engineering and Design 237, 289-299.

Stepanishen, P.R., 1997. Transient vibratory response of fluid-loaded structures using convolution integral equations. Journal of Acoustical Society of America 101, 1877-1889.

Stokes, G.G., 1851. On the effect of the internal friction of fluids on the motion of pendulums. Transactions of the Cambridge Philosophical Society 9, 8-106.

Stuart, J.T., 1963. Unsteady boundary layers. In: Rosenhead, L. (Ed.), Laminar Boundary Layers. Clarendon Press, Oxford, pp. 349-408.

Wang, C.Y., 1968. On high-frequency oscillatory viscous flows. Journal of Fluid Mechanics 32, 55-68. 